\title{
On the variety of particle accumulation structures under the effect of gjitters
}

\author{
Marcello Lappa ${ }^{1}$ \\ ${ }^{1}$ Telespazio, Via Gianturco 31 - Napoli, 80046 - Italy, marlappa@unina.it
}

Abstract: The present analysis extends the author's earlier work (Lappa, Phys. Fluids 25(1) and Chaos 23(1)) on the properties of patterns formed by the spontaneous accumulation and ordering of solid particles in certain types of flow (with a toroidal structure and a traveling wave propagating in the azimuthal direction) by considering the potential impact of "vibrations" (gjitters) on such dynamics. It is shown that a kaleidoscope of possible variants exist whose nature and variety calls for a concerted analysis using the tools of computational fluid-dynamics in synergy with dimensional arguments and existing theories on the effect of periodic accelerations on fluid systems. A possible categorization of the observed phenomena is introduced according to the type and scale of "defects" displayed by the emerging particle aggregates with respect to unperturbed (vibrationless) conditions. It is shown that the resulting degree of "turbulence" depends essentially on the direction $(\phi)$, amplitude $(\gamma)$ and frequency $(\varpi)$ of the applied inertial disturbance. A range of amplitudes and frequencies exist where the formation of recognizable particle structures is prevented. A quantitative map (in the $\gamma-\varpi$ plane) for their occurrence is derived with the express intent of supporting the optimization of future experiments to be performed in space.

\section{Introduction}

Dispersed multiphase flows are commonly encountered in a variety of natural and technological applications. The study of such phenomena is wide ranging (see, e.g., Zohdi, 2007; Zaichik et al., 2008) and touches various scientific fields, including (but not limited to) environmental phenomena, thermal and materials engineering, crystal growth, life science and many other related topics.

It is a well-known fact that drops and bubbles dispersed within a surrounding immiscible phase can migrate as a result of gravity and/or forces of surface-tension-driven origin and accumulate in some specific regions of the liquid (typically at the bottom or at the top of the container due to sedimentation or the opposite effect, or in regions of higher temperature due to surface-tensiondriven effects, see, e.g., Esmaeeli and Tryggvason, 1998; Esmaeeli, 2005, Lappa, 2005 and 2006 and references in all these works). Another well-documented phenomenon that can affect a dispersed phase (it requires neither an external force field nor surface-tension-driven effects) is inertial clustering, i.e. concentration of particles into narrow bands due to their inertia.

Several studies pertaining to this line of inquiry have shown both theoretically and experimentally that the presence of a particle with nonzero mass and size modifies the flow locally and, therefore, 
the motion of such a particle in the fluid typically differs from that of an ideal tracer, which would simply follow the local velocity of the flow.

The main overarching principle governing such dynamics (see, e.g., Balkovsky et al., 2001) is that, because of finite particles' inertia, the velocity field of a set of spatially distributed particles is compressible (even if the fluid flow is incompressible). It has been argued that for particles with solid/fluid density ratio $\xi \neq 1$ this property can produce fluctuations of concentration significant and can, hence, support mechanisms for particle concentration and ordering. This preferential accumulation can still occur if the particle dynamics is chaotic (which is often the case in most of practical applications; in general, in fact, the dynamics of a small, but finite-size, spherical particle transported by a fluid flow obeying equations of motion that are inherently dissipative, can be chaotic even with a flow field that is simply time periodic).

There is a long tradition of studies on this subject, essentially focusing on the role played by particle inertia and viscous drag (in association with regions of high shear present in the fluid and a more or less pronounced degree of turbulence of the considered velocity field) in determining phenomena of particle demixing and spontaneous association in specific regions of the physical space.

As an example, Benczik et al. (2002) examined nonturbulent, low Reynolds number flows, with Re on the order of hundreds. They demonstrated analytically that small deviations in particle trajectories tend to grow exponentially in time at a rate given by the largest positive Lyapunov exponent of the dynamics that, in turn, is influenced by the inertia parameters. They expressly showed, however, that since the inertial motion is dissipative, there are situations in which the phase-space contraction rate can become positive even in incompressible flows, thereby opening the possibility of having attractors in the dynamics (such attractors exist in the full phase space as invariant sets, but what we observe in practice is always a projection of these sets onto the configuration space, i.e. the physical space).

As additional examples, Maxey et al. (1997) and Saw et al. (2008) reported numerical and experimental evidence, respectively, of spatial clustering of dense particles in high-Re homogenous, isotropic turbulence. The dissipation-scale clustering was found to become stronger for increasing values of the Stokes number. Owing to the large Reynolds numbers considered, in particular, a broad scaling range of particle clustering due to turbulent mixing was identified (by which they distinguished clustering of inertial nature from that due to the mixing of fluid particles).

Some interesting criteria to predict the exact location of inertial particle association in threedimensional steady or two-dimensional time-periodic flows were provided by Sapsis and Haller (2010) (and enriched later by Haller and Sapsis, 2012 through specific considerations on the behaviour of the smallest finite-time Lyapunov exponent). In these studies the particles were found to accumulate due to their finite size on attracting inertial Lagrangian coherent structures (ILCS) that are smooth deformations of invariant tori.

This line of inquiry has been recently expanded by considerations on the role played in these dynamics by potential mechanisms of phase-locking between traveling fluid-dynamic disturbances (propagating in the considered fluid medium in the form of waves of given wavenumber and frequency), on one side, and the typical particle turnover frequency (the characteristic frequency 
taken by the particle to follow a given closed path, Schwabe et al et al. 2007, Pushkin et al. 2011 or its angular spin, Lappa, 2013a,b) on the other side.

This new branch of investigation has specially focused on the dynamics of particle accumulation and ensuing patterning phenomena in 3D fluid systems with axisymmetry (the motivation behind such a specific choice lying essentially in the well-known homogeneity of conditions to be imposed in the azimuthal dimension for such axisymmetric fluid systems, which can be realized easily in experiments and numerical simulations, Busse et al., 1998).

It has been shown that if specific conditions are satisfied, particles, initially uniformly spaced in the liquid, are allowed to demix and form an "apparently solid thread" (generally referred to as a particle accumulation structure - PAS), which rotate at an angular velocity equal to the angular frequency of the thermofluid-dynamic disturbance. The fascinating strictly one-dimensional nature of the resulting shapes distinguishes this case from similar phenomena observed previously for other categories of flow (the reader may consider Lappa, 2012 for an exhaustive historical perspective on this subject).

While the bizarre variety of PAS still presents a challenge for the existing theories (Lappa, 2013b), their ubiquity suggests that their formation is a robust process (their occurrence has been demonstrated for both Marangoni flow and Rayleigh-Bénard convection, and most recently also in situations where the conditions for their existence are created artificially, Kleckner and Irvine, 2013). This means we now are in a situation where the study of PAS phenomena has reached a sort of maturity, in the sense that numerical and experimental techniques are in position to convey valuable and relevant information concerning most of the questions we may ask on the problem. It is just with this spirit that a set of experiments to be performed in space has been planned and approved by major space agencies for execution onboard the International Space Station (ISS) (Shevtsova et al., 2008).

In the present study we continue our computational investigation into the topic of particle accumulation in Marangoni flow as provided by a liquid bridge in microgravity conditions with suitable liquid, aspect ratio and applied temperature difference.

While the earlier work (Lappa, 2013a) focused on the mechanism of spontaneous accumulation of solid particles alone, we now aim at identifying and quantifying some effects which may alter or even prevent such a process. More specifically, we refer to the phenomena that arise through the interaction of the classical PAS with inertial perturbations potentially entering the considered fluid system in the form of vibrations or small body accelerations.

This subject is motivated by several factors. From a theoretical standpoint it is unknown whether the physical process leading to the formation of PAS persists as such disturbances gain importance (and if it does, whether or not the properties of the resulting PAS depend on their strength). It is unknown whether PAS formation is enhanced or adversely affected depending on disturbance amplitude, frequency and direction.

The second motivation is purely technical. As mentioned before, due to typical limitations imposed by terrestrial gravity, experiments are being planned for execution onboard the ISS (Shevtsova et al., 2013). Albeit this environment makes possible extended liquid/gas interfaces (of the order of some 
centimeters) and the avoidance of undesired buoyancy effects, it, however, suffers from some degree of residual accelerations. Among these, the so-called "g-jitters" have relatively high frequency content. These perturbations typically come from pulsating or impulsive external loads, such as motor firings during maneuvers, crew activity, station motorized equipments, station moving elements, space debris impacts, and the station structural elastic response to these loads. In practice, they include all of the periodic time-dependent accelerations that can be approximated by sinusoidal functions. For the reasons highlighted above, the eventual benefit of microgravity for studying PAS must be carefully assessed.

\section{Mathematical Models and Methods}

A full understanding of the problem considered here requires modeling it in a consistent way on many levels simultaneously. The first of these levels involves a representation of the inertial disturbances (the gjitters, see Sect. 2.1). The related mathematical formalism must be sufficiently general to allow the consideration of a wide class of potential disturbances, and, at the same time, simple enough not to increase excessively the complexity of the resulting governing equations. These, in turn, must allow simulation of the flow in the liquid as completely as possible (Sect. 2.2 and 2.3). A third level of complexity in such a modeling hierarchy is given by the simulation of the solid particle motion (Sect. 2.4). Such a simulation brings problems of its own, including the issue of discerning whether it is a good representation of reality (Sect. 2.5 and 2.6).

An elementary illustration of these topics is elaborated in the following (it is "elementary" because our derivations and discussion are obtained by direct and straightforward manipulations of the equations of motion, often in the simplest case that will illustrate the relevant overarching principle).

\subsection{Gjitters}

In this section we will merely touch on this subject. It would otherwise occupy the entire discussion (see, e.g., Alexander, 1990; Monti et al., 1998 and 2001).

Perturbations induced in a fluid by a sinusoidal displacement of a fluid system along a given direction ( $\underline{\hat{n}}$ is the related unit vector)

$$
\underline{s}(t)=b \sin (\omega \mathrm{t}) \underline{\hat{n}}
$$

where $\mathrm{b}$ is the amplitude and $\omega=2 \pi \mathrm{f}$ ( $\mathrm{f}$ is the frequency), induce an acceleration:

$$
\underline{g}(t)=g_{\omega} \sin (\omega \mathrm{t}) \underline{\hat{n}}
$$

where $g_{\omega}=\mathrm{b} \omega^{2}$. 
which means vibrating a system with frequency $\mathrm{f}$ and displacement amplitude $\mathrm{b}$ corresponds to a sinusoidal gravity modulation with the same frequency and acceleration amplitude $b \omega^{2}$ and vice versa (accordingly, hereafter the terms "gravity modulation", "periodic acceleration", "system vibration" and g-jitter will be used as synonyms).

Equation (2) represents the idealized situation of a single frequency (generally referred to as "monochromatic" disturbance). In reality, however, an effective microgravity environment is given by the superposition of disturbances with different amplitudes and frequencies (multifrequency or multicomponent spectrum) and, eventually, distinct directions. For simplicity here we consider essentially monochromatic disturbances, allowing, however, their amplitude and frequency to span a significantly wide range (some useful models/results for the more realistic case of coloured noise will be elaborated/discussed in Sect. 4.3).

\subsection{Nondimensional balance equations}

The non-dimensional balance equations can be obtained from scaling the co-ordinates by the axial system extension (L) and the velocity components by the energy diffusion velocity $V_{\alpha}=\alpha / L$ where $\alpha$ is the fluid thermal diffusivity; the scales for time and pressure are, respectively, $\mathrm{L}^{2} / \alpha$ and $\rho \alpha^{2} / \mathrm{L}^{2}$, where $\rho$ is the fluid density. The temperature is measured with respect to its initial value $\overline{\mathrm{T}}_{\mathrm{m}}$ and is made nondimensional as $\mathrm{T}=\left(\overline{\mathrm{T}}-\overline{\mathrm{T}}_{\mathrm{m}}\right) / \Delta \mathrm{T}$ where $\Delta \mathrm{T}$ is the imposed temperature gradient. In such a framework and using the Boussinesq approximation to account for the presence of body accelerations as defined in Sect. 2.1, the continuity and momentum equations simply read:

$\underline{\nabla} \cdot \underline{V}=0$

$\frac{\partial \underline{V}}{\partial t}=-\underline{\nabla} p-\underline{\nabla} \cdot[\underline{V} \underline{V}]+\operatorname{Pr} \nabla^{2} \underline{V}+\operatorname{Pr} \frac{b \omega^{2} \beta_{T} \Delta T L^{3}}{v \alpha} T \sin \left(\frac{L^{2} \omega}{\alpha} t\right) \underline{\hat{n}}$

where $\frac{b \omega^{2} \beta_{T} \Delta T L^{3}}{v \alpha}=R a_{\omega}$ can be regarded as a variant of the classical Rayleigh number with the steady acceleration being replaced by the amplitude of the considered monochromatic periodic acceleration ( $\beta_{\mathrm{T}}$ is the thermal expansion coefficient).

Remarkably, in this form the equation allows the treatment of the problem in terms of four independent nondimensional parameters only, where the first is the well-known Prandtl number $(\mathrm{Pr}=v / \alpha$ where $v$ is the fluid kinematic viscosity) and the others are the nondimensional frequency $(\varpi)$, the nondimensional acceleration amplitude $(\gamma)$ and buoyancy factor $(\beta)$ defined as:

$\varpi=\frac{\omega L^{2}}{\alpha}$ 
$\gamma=\frac{b \omega^{2} L^{3}}{\alpha^{2}}$

$\beta=\left(\beta_{T} \Delta T\right)$

Accordingly, the momentum equation can be cast in compact form as:

$\frac{\partial \underline{V}}{\partial}=-\underline{\nabla} p-\underline{\nabla} \cdot[\underline{V} \underline{V}]+\operatorname{Pr} \nabla^{2} \underline{V}+\gamma \beta T \sin (\varpi t) \underline{\hat{n}}$

where, obviously $\operatorname{Ra}_{\omega}=\gamma \beta / \operatorname{Pr}$.

This equation must be supplemented with the energy balance equation in nondimensional form that with the considered reference units reads:

$\frac{\partial T}{\partial t}+\underline{\nabla} \cdot[\underline{V} T]=\nabla^{2} T$

\subsection{The Geometry}

Since most of existing studies have considered PAS structures in liquid bridges (with the supercritical Marangoni flow in the so-called rotating regime, see (Schwabe et al., 2006, 2007; Tanaka et al., 2006; Ueno et al., 2008), the starting point of our analysis is represented by such a geometry (Figure 1).

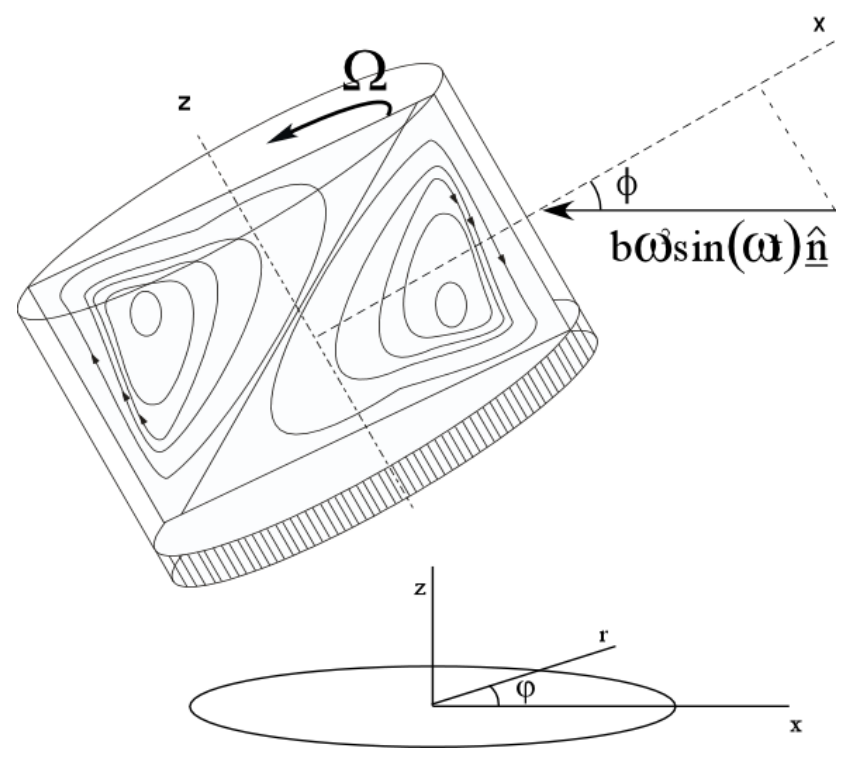

Figure 1: Liquid bridge and applied gjitters. The $\Omega$ symbol indicates the angular frequency of the hydrothermal wave propagating in the azimuthal direction. The coordinate system used to characterize the flow inside the liquid bridge is also shown on the bottom of the figure. 
A drop of liquid with cylindrical free liquid/gas interface is held between two disks of diameter D placed L apart, as shown in Figure 1. The considered gjitters are contained in the xz plane and form an angle $\phi$ with the $\mathrm{x}$ axis $(0 \leq \phi \leq \pi / 2)$.

Moreover, circumstances are considered for which the supercritical Marangoni flow permits the formation of PAS. Accordingly, we focus on the following well known test case (Melnikov et al., 2011; Lappa, 2013a,b): a liquid bridge with aspect ratio $A=0.34$ (A being the height to diameter ratio) delimited by solid horizontal disks (one at $\mathrm{z}=0$ cooled, the other at $\mathrm{z}=1$ heated, adiabatic conditions on the liquid/gas interface). The considered values of the Prandtl and Marangoni numbers are $\operatorname{Pr}=8$ and $\mathrm{Ma}=2.06 \times 10^{4}$, respectively (Ma defined as $\sigma_{\mathrm{T}} \Delta \mathrm{TL} / \mu \alpha$ where $\sigma_{\mathrm{T}}$ is the surface tension derivative with respect to temperature and $\mu=\rho v$ the dynamic viscosity). Moreover, no steady residual gravity is considered $(\mathrm{Ra}=0)$ and the liquid/gas interface is considered undeformable under the assumption that $\mathrm{Bo}=\rho \mathrm{g}_{\omega} \mathrm{L}^{2} / \sigma<<1$ and $\mathrm{Ca}=\rho v^{2} / \sigma \mathrm{L}<<1$ and that (as confirmed by several studies) the amplitude of interface oscillations is very small.

Indicating the velocity components in the axial (z), radial (r) and azimuthal $(\varphi)$ directions with $\mathrm{u}, \mathrm{v}$ and $\mathrm{w}$, respectively, and projecting the governing equations along these directions yields in the (fixed) laboratory frame:

$\frac{\partial u}{\partial z}+\frac{\partial v}{\partial r}+\frac{1}{r}\left(v+\frac{\partial w}{\partial \varphi}\right)=0$

$\frac{\partial u}{\partial t}=-\frac{\partial p}{\partial z}-(\underline{V} \cdot \underline{\nabla} \underline{V})_{u}+\operatorname{Pr}(\Delta \underline{V})_{u}+\gamma \beta T \sin (\varpi t) \sin (\phi)$

$\frac{\partial v}{\partial}=-\frac{\partial p}{\partial r}-(\underline{V} \cdot \underline{\nabla} \underline{V})_{v}+\operatorname{Pr}(\Delta \underline{V})_{v}+\gamma \beta T \sin (\varpi t) \cos (\phi) \cos (\varphi)$

$\frac{\partial w}{\partial}=-\frac{1}{r} \frac{\partial p}{\partial \varphi}-(\underline{V} \cdot \underline{\nabla} \underline{V})_{w}+\operatorname{Pr}(\Delta \underline{V})_{w}+\gamma \beta T \sin (\varpi t) \cos (\phi) \sin (\varphi)$

where $p$ is the pressure and

$$
\begin{aligned}
& (\underline{V} \cdot \underline{\nabla} \underline{V})_{u}=\left(u \frac{\partial u}{\partial z}+v \frac{\partial u}{\partial r}+\frac{w}{r} \frac{\partial u}{\partial \varphi}\right) \\
& (\underline{V} \cdot \underline{\nabla} \underline{V})_{v}=\left(u \frac{\partial v}{\partial z}+v \frac{\partial v}{\partial r}+\frac{w}{r} \frac{\partial v}{\partial \varphi}-\frac{w^{2}}{r}\right) \\
& (\underline{V} \cdot \underline{\nabla} \underline{V})_{w}=\left(u \frac{\partial w}{\partial z}+v \frac{\partial w}{\partial r}+\frac{v w}{r}+\frac{w}{r} \frac{\partial w}{\partial \varphi}\right) \\
& (\Delta \underline{V})_{u}=\left(\frac{\partial^{2} u}{\partial z^{2}}+\frac{\partial^{2} u}{\partial^{2}}+\frac{1}{r} \frac{\partial u}{\partial r}+\frac{1}{r^{2}} \frac{\partial^{2} u}{\partial \varphi^{2}}\right)
\end{aligned}
$$




$$
\begin{aligned}
& (\Delta \underline{V})_{v}=\left(\frac{\partial^{2} v}{\partial z^{2}}+\frac{\partial^{2} v}{\partial r^{2}}-\frac{v}{r^{2}}+\frac{1}{r} \frac{\partial}{\partial r}+\frac{1}{r^{2}} \frac{\partial^{2} v}{\partial \varphi^{2}}-\frac{2}{r^{2}} \frac{\partial w}{\partial \varphi}\right) \\
& (\Delta \underline{V})_{w}=\left(\frac{\partial^{2} w}{\partial z^{2}}+\frac{\partial^{2} w}{\partial r^{2}}+\frac{1}{r} \frac{\partial w}{\partial r}-\frac{w}{r^{2}}+\frac{1}{r^{2}} \frac{\partial^{2} w}{\partial \varphi^{2}}+\frac{2}{r^{2}} \frac{\partial}{\partial \varphi}\right) \\
& \frac{\partial T}{\partial t}=-\left(\frac{\partial u T}{\partial z}+\frac{\partial T}{\partial r}+\frac{v T}{r}+\frac{1}{r} \frac{\partial w T}{\partial \varphi}\right)+\left(\frac{\partial^{2} T}{\partial z^{2}}+\frac{\partial^{2} T}{\partial^{2}}+\frac{1}{r} \frac{\partial T}{\partial r}+\frac{1}{r^{2}} \frac{\partial^{2} T}{\partial \varphi^{2}}\right)
\end{aligned}
$$

For $\mathrm{t}>0$, the boundary conditions at the free surface are the well known Marangoni conditions accounting for shear stress balance and the kinematic condition of stream surface (see, e.g., Savino et al., 2003):

$$
\begin{aligned}
& \frac{\partial \mathrm{u}}{\partial r}(\mathrm{z}, r=R / L, \varphi, t)=-M a \frac{\partial T}{\partial \mathrm{z}}(\mathrm{z}, r=R / L, \varphi, t) \\
& v(\mathrm{z}, \mathrm{r}=\mathrm{R} / \mathrm{L}, \varphi, \mathrm{t})=0 \\
& r \frac{\partial w}{\partial r}(z, r=R / L, \varphi, t)-w(z, r=R / L, \varphi, t)=-M a \frac{\partial T}{\partial \varphi}(z, r=R / L, \varphi, t)
\end{aligned}
$$

The other kinematic conditions to be imposed on the walls simply reflect the well-known no-slip and impermeability properties of solid boundaries $(\mathrm{u}=\mathrm{v}=\mathrm{w}=0)$. We do not report them here explicitly for the sake of brevity (and given their extreme simplicity, see, e.g., Lappa and Savino, 2002 or Lappa et al., 2003). For problem closure, such conditions, however, have to be supplemented with those for the energy equation. As outlined before, the lower $(\mathrm{z}=0)$ and upper $(\mathrm{z}=1)$ walls of the domain are assumed here to be at uniform and constant (nondimensional) temperatures $\mathrm{T}_{0}=1$ and $\mathrm{T}_{1}=0$ respectively, while the liquid/gas interface is adiabatic, i.e. $\partial \mathrm{T} / \partial \mathrm{r}=0$ is imposed there.

\subsection{The Dispersed Phase}

Given the considered topic, the present problem may be regarded as a typical example of situations in which one witnesses an interplay between large-scale (liquid bridge and its supercritical Marangoni flow) and small-scale entities and processes (particles and their motion under the influence of large-scale flow and periodic accelerations), which, in general, require a multiscale approach. Accordingly, two models have to be defined to close the problem properly from a mathematical point of view, one referring to the nonlinear nature of a large-scale flow and the second dealing with issues strictly related to the displacement of particles in time and associated mechanisms. 
In other words, this means that some model diversification must be introduced on the basis of the desired scale length, i.e. according to the level of detail required by the considered phenomena, these being: "bulk flow" or "particle motion".

The former case has been covered by Sect. 2.3. The latter requires a treatment which depends on the specific situation involved and especially the "nature" of the dispersed phase.

In principle, many approaches have been developed over the years to deal with situations such as this, especially for cases where there are two distinct fluid phases (liquid-liquid or gas-liquid).

In general, all of these techniques can be classified under three main categories according to the intrinsic nature of the philosophy used to define their main features: capturing (also known as moving grid or Lagrangian approach, see Uchiyama, 2011 and references therein), Eulerian (also known as fixed-grid approach, see, e.g., Lappa et al., 2004; Lappa, 2005, 2006; Bothe et al., 2011) and combined "hybrid" techniques (e.g., Homma et al., 2011; Mark et al., 2011).

In the present case, since the dispersed phase is constituted of small particles which undergo neither dimensional changes, nor shape modification (they are assumed to be undeformable), there is no doubt that, amongst the possible strategies reviewed above, the most relevant model should be introduced as a "hybrid" formulation. More precisely, one may imagine to use an Eulerian approach to determine the large-scale flow (eq. 8-13) and then capture particle motion with a Lagrangian method.

Despite this obvious way of thinking, the resulting algorithm may not be straightforward as one may imagine if some additional simplifying assumptions are not considered. These additional conditions concern, in particular, the influence of particle motion on the large-scale flow and the particle-particle interplay (Derksen and Eskin, 2011; Balboa Usabiaga et al., 2013).

To fix these issues, like earlier studies (Melnikov et al., 2011; Kuhlmann and Muldoon, 2012), our investigation targets the dilute regime, i.e. it is assumed that the effect of the particles onto the fluid, as well as the interaction among particles are negligible (in the presence of vibrations, as shown by several authors, this is permissible if the concentration of the dispersed phase in the flow is small, i.e. if the distance of a particle from others is much larger than their diameter; see, e.g. Langbein, 1991; Kozlov et al., 2006; and Hassan et al. 2006b, see, in particular, Figure 4 in their work). Hence, the resulting method may be regarded as a special case of a single-region formulation in which the dispersed phase experiences lagrangian transport without influencing the large-scale flow (in this regard it may be seen as a variant of the so-called "population methods", see, e.g., Zinchenko, 1994, for which, however, the dispersion of spheres is dilute rather than concentrated).

Particles are treated as isolated, microscopic quantities compared with field variables, but their motion is tracked by using an appropriate equation taking into account inertia (including the added mass effect) and viscous drag forces depending on particle specific shape (see, e.g., Maxey and Riley, 1983; Michaelides, 1997; Babiano et al., 2000). Such a philosophy allows a simple and efficient treatment of the problem from a computational point of view and has been applied successfully to situations in which the physical phenomena of interest have a large spatial scale with respect to the average particle size. For the specific case of PAS dynamics, most of earlier studies on this subject (Pushkin et al. 2011; Lappa 2013a,b) used the so-called "inertial equation" (leading 
to several advantages in terms of computational efficiency); here, however, given the intrinsic degree of unsteadiness of the velocity field (due to the presence of gjitters), we resort to a more complete variant :

$$
\begin{aligned}
& \frac{d u_{\text {partcl }}}{d t}=\frac{1}{\xi+1 / 2}\left[-\frac{\operatorname{Pr}}{S t}\left(u_{\text {partcl }}-u\right)+\frac{3}{2} \frac{d u}{d t}+\frac{3}{2}(\underline{V} \cdot \underline{\nabla} \underline{V})_{u}-\right. \\
& \left.\sqrt{\frac{9}{2 \pi} \frac{\operatorname{Pr}}{S t}} \int_{o}^{t} \frac{1}{\sqrt{t-\widetilde{t}}} \frac{d}{d \widetilde{t}}\left(u_{\text {part }}-u\right) d \widetilde{t}\right]+\frac{\xi-1}{\xi+1 / 2} \gamma \sin (\varpi t) \sin (\phi) \\
& \frac{d v_{\text {partcl }}}{d t}=\frac{1}{\xi+1 / 2}\left[-\frac{\operatorname{Pr}}{S t}\left(v_{\text {partcl }}-v\right)+\frac{3}{2} \frac{d v}{d t}+\frac{3}{2}(\underline{V} \cdot \underline{\nabla V})_{v}-\right. \\
& \left.\sqrt{\frac{9}{2 \pi} \frac{\operatorname{Pr}}{S t}} \int_{o}^{t} \frac{1}{\sqrt{t-\widetilde{t}}} \frac{d}{d \widetilde{t}}\left(v_{\text {part }}-v\right) d \widetilde{t}\right]+\frac{\xi-1}{\xi+1 / 2} \gamma \sin (\varpi t) \cos (\phi) \cos (\varphi) \\
& \frac{d w_{\text {partcl }}}{d t}=\frac{1}{\xi+1 / 2}\left[-\frac{\operatorname{Pr}}{S t}\left(w_{\text {partcl }}-w\right)+\frac{3}{2} \frac{d w}{d t}+\frac{3}{2}(\underline{V} \cdot \underline{\nabla} \underline{V})_{w}-\right. \\
& \left.\sqrt{\frac{9}{2 \pi} \frac{\operatorname{Pr}}{S t}} \int_{o}^{t} \frac{1}{\sqrt{t-\widetilde{t}}} \frac{d}{d \widetilde{t}}\left(w_{\text {part }}-w\right) d \tilde{t}\right]+\frac{\xi-1}{\xi+1 / 2} \gamma \sin (\varpi t) \cos (\phi) \sin (\varphi)
\end{aligned}
$$

where $\xi$ is the ratio of the particle to the fluid density and $\underline{V}_{\text {part }}\left(\mathrm{u}_{\text {part }}, \mathrm{v}_{\text {part }}, \mathrm{w}_{\text {part }}\right)$ and $\underline{V}$ are the particle and fluid velocity, respectively (the Maxey-Riley equation).

The underlying assumptions on which this approach is based are, therefore, a perfectly spherical shape of the solid particles and a very small value $(<<1)$ of the particle to fluid system characteristic size ratio $\widetilde{R} / L$ (where $\widetilde{R}$ is the solid particle radius). Moreover, the so-called particle relaxation (response) time must be much less than the characteristic time scales of the considered flow (Michaelides, 1997; Babiano et al., 2000).

The particle relaxation time $(\tau)$ can be defined as:

$$
\tau=\frac{2}{9} \frac{\widetilde{R}^{2}}{v}
$$

As the characteristic flow time scale of the Marangoni flow we assume here (as in other studies, see, e.g., Pushkin et al., 2011):

$$
\tau_{\text {flow }}=\frac{L^{2}}{v}
$$

A properly defined time scale for the vibration-induced flow can be introduced as: 
$\tau_{v}=\frac{2 \pi}{\omega}$

Nondimensional parameters accounting for the relative importance of the particle relaxation time and these characteristic times, in turn, can be defined as (see Hjelmfelt and Mockros, 1966; Coimbra and Rangel, 2001):

$$
\begin{aligned}
& S t=\frac{\tau \nu}{L^{2}}=\frac{\eta}{\xi-1} \operatorname{Pr} \\
& S t_{v}=\frac{\tau \omega}{2 \pi}=\frac{1}{2 \pi} \frac{\eta \varpi}{\xi-1}
\end{aligned}
$$

They are generally referred to as the Stokes number and vibrational Stokes number, respectively, where

$$
\eta=\frac{\tau \alpha}{L^{2}}(\xi-1)
$$

is the general parameter that can be used (see, e.g., Pushkin et al., 2011, Lappa, 2013a) to account for the combined effect of particle inertia and drag $\left(\eta=\mathrm{O}\left(10^{-5}\right)\right.$ for the conditions considered in the present work).

The limit $\mathrm{St}_{\mathrm{v}} \rightarrow 0$ represents the condition for which a fixed viewer would see the particle moving with the same amplitude as the imposed vibration, i.e. particles "frozen" in the vibrations field, the opposite limit $\mathrm{St}_{\mathrm{v}}>>1$, representing situations in which particle would lose the capability to follow any velocity change in the flow (Simic-Stefani et al., 2006). Both dimensionless parameters St and $\mathrm{St}_{\mathrm{v}}$, must be sufficiently small $(<<1)$ to make eq. (14) valid.

The following conditions, therefore, must be satisfied:

$\eta<<(\xi-1) / \operatorname{Pr}$

and, owing to the presence of gjitters with their characteristic time scale $2 \pi \omega^{-1}$,

$\varpi<<2 \pi(\xi-1) / \eta$

As for the case considered in the present work $(\operatorname{Pr}=8)$ PAS are formed for $\xi=1.85$ and $\eta \cong 10^{-5}$, eq. (19a) is implicitly satisfied. Equation (19b), however, puts a constraint on the maximum frequency that can be considered, which for the indicated values of $\operatorname{Pr}, \xi$ and $\eta$ has to be $\varpi<\mathrm{O}\left(10^{6}\right)$. Here, in particular, to be on a safe side the maximum $\varpi$ is fixed to $\varpi=5 \times 10^{4}$ (that is approximately two order of magnitudes smaller than the maximum theoretically allowed value). 
In addition to the above restrictions, however, conditions must be also satisfied for which the socalled history term (the Basset force, given by the integral contribution in eq. 14) can be neglected. The inclusion of this term in the model makes the particle equation non-explicit in the velocity or acceleration, leading to a cumbersome and computationally time-consuming numerical problem (intractable for the extensive number of particles considered in this work).

According to the criteria provided by Hjemfelt and Mocros (1966), the effect of this term should be neglected a priori when the Stokes number $\left(\mathrm{St}\right.$ and $\mathrm{St}_{\mathrm{v}}$ ) satisfies the condition $<2 / 9 \xi^{1 / 2} \cong 3 \times 10^{-1}$.

An evaluation of $\mathrm{St}$ and $\mathrm{St}_{\mathrm{v}}$ with the present choice of parameters shows that this criterion is met with $\mathrm{St} \cong 10^{-4}$ and $\mathrm{St}_{\mathrm{v}} \cong 10^{-1}$ (assuming for $\varpi$ the worst case, i.e. its largest considered value $5 \times 10^{4}$ ).

In order to understand whether the maximum tolerable value of $\varpi$ has to be further constrained or not, we have also considered the more recent investigations by Vojir and Michaelides (1994) and Coimbra and Rangel (2001), who conducted excellent numerical studies on this subject by computing the effect of the history term on the particle motion in sinusoidally varying velocity fields.

For a frequency corresponding to $\varpi=4.6 \times 10^{5}$, Vojir and Michaelides (1994) found the history term to have a significant effect on particle dynamics for $1.43<\xi<500$. At smaller frequencies and assuming a density ratio relatively close to that considered here, these authors concluded that the effect of the Basset term could be neglected, provided the nondimensional angular frequency of the sinusoidally varying velocity field was $<5 \times 10^{3}(<0.1$ with their reference units, see Figure 2a of their work). These conclusions also agree with the analysis by Druzhinin and Ostrovsky (1994) and Coimbra and Rangel (2001). The latter authors, in particular, found the relative importance of history force for $\xi \neq 1$ to be particularly significant for $\mathrm{St}_{\mathrm{v}} \cong 0.3$ (more specifically, it was found to attain a maximum value for a dimensional frequency approaching the value $9 v / \widetilde{R}^{2}$ and displacement amplitudes $\mathrm{b}$ smaller than the radius $\widetilde{R}$ of the particle, conditions that using the present reference units would read: $\varpi \cong 2 \mathrm{PrSt}^{-1}=1.6 \times 10^{5}$ and $\left.\gamma / \varpi^{2} \leq(9 \mathrm{St} / 2)^{1 / 2} \cong 2 \times 10^{-2}\right)$. Albeit the importance of the Basset term was observed to decrease rapidly as the value of $\mathrm{St}_{\mathrm{v}}$ approached 0 for all values of $\xi$ (as confirmed later in the experiment by Coimbra et al., 2004), however, they found the ratio of the Basset force to the overall force experienced by the particle (virtual mass + stokes drag + history term) to be $\cong 0.2$ (see Figure 1 of their work) for a value of their control parameter $S=0.06$, which corresponds to the present $\varpi \cong 10^{4}$.

As the studies by Vojir and Michaelides (1994) and Coimbra and Rangel (2001), both indicate that the Basset term may play a potentially significant role for the conditions considered in the present study when $\varpi>5 \times 10^{3}$, it has been therefore deemed necessary to assess expressly its influence on PAS for relatively high frequencies (the results of this study will be discussed in Sect. 3).

\subsection{Solution Method and Validation Study}

Balance equations (8-13) have been solved numerically by a time-explicit finite-difference method (primitive-variable method) based on a cylindrical mesh and a staggered collocation of fluiddynamic variables. Forward differences in time and central-differencing schemes in space (second 
order accurate) have been used to discretize the energy and momentum governing equations. The related solution strategy is not discussed here, the interested reader being referred to various books and articles in the literature for an exhaustive treatment (for the implementation of this method on parallel machines, the reader may consider, e.g., Lappa and Savino, 1999; Lappa, 2003a and 2004). The above statement, however, does not apply to the part concerning the computation of PAS with equations (14), which was not validated in earlier studies of the present author (both Lappa, 2013a and 2013b, in fact, were based on a simplified version of the Maxey-Riley equation, the so-called inertial equation). Albeit it has been shown that the inertial equation can provide significant advantages in predicting the asymptotic dynamics of inertial particles, however, that equation (in which $d \underline{V}_{\text {part }} / d \mathrm{t}$ is assumed to be 0 ) should not be used in potentially strongly unsteady situations like those considered here, especially if one is interested in capturing the overall evolutionary path taken by the considered system in approaching an asymptotic state (provided this asymptotic state exists).

Prior to embarking in the description of the validation study, let us recall for the convenience of the reader, that when the temperature difference is small, a steady vortex ring is established inside the liquid bridge in which the rotating fluid takes on a toroid or doughnut shape. This ring consists of surface flow moving from the hot side to the cold side of the outer surface and a return flow in the interior of the liquid bridge. Increasing the temperature difference above a certain threshold produces flow patterns that vary with time (supercritical Marangoni flow, see Figure 2a). For transparent liquids, adding tracer particles to the liquid reveals the main characteristics of such a flow (see, e.g., Shevtsova et al, 2011 and references therein).

With specific regard to PAS, viewing the flow through the transparent top rod (e.g., a sapphire crystal), such a structure (PAS) looks like a "windmill", with m blades (where $m$ depends on the aspect ratio of the liquid bridge) rotating at a constant speed. But the rotation is an illusion because the particles follow the flow, which is essentially toroidal. In fact, it is the three-dimensional pattern that rotates, not the liquid, and the individual particles organize themselves into what appears to be a rotating solid thread.

TABLE I: Comparison with the results of Melnikov et al. (2011), liquid bridge with aspect ratio (height/diameter) $=0.34, \operatorname{Pr}=8\left(\mathrm{NaNO}_{3}\right), \mathrm{Ma}=20600, \xi=1.85$ (no gjitters considered).

\begin{tabular}{|c|c|c|c|c|}
\hline Ref & $\begin{array}{c}\text { Grid } \\
\left(\mathrm{N}_{\mathrm{Z}} \mathrm{xN}_{\mathrm{r}} \mathrm{xN}_{\varphi}\right)\end{array}$ & $\begin{array}{l}\text { Mode } \\
\mathrm{m}\end{array}$ & $\begin{array}{l}\text { Nondimensional } \\
\text { Angular frequency of } \\
\text { the hydrothermal wave } \\
\qquad \Omega=2 \pi \mathrm{f} / \mathrm{m}\end{array}$ & PAS \\
\hline $\begin{array}{l}\text { Melnikov et al. } \\
(2011)\end{array}$ & $40 \times 40 \times 32$ & 3 & 73.3 & Yes \\
\hline Present & $32 \times 40 \times 40$ & 3 & 71.4 & Yes \\
\hline
\end{tabular}



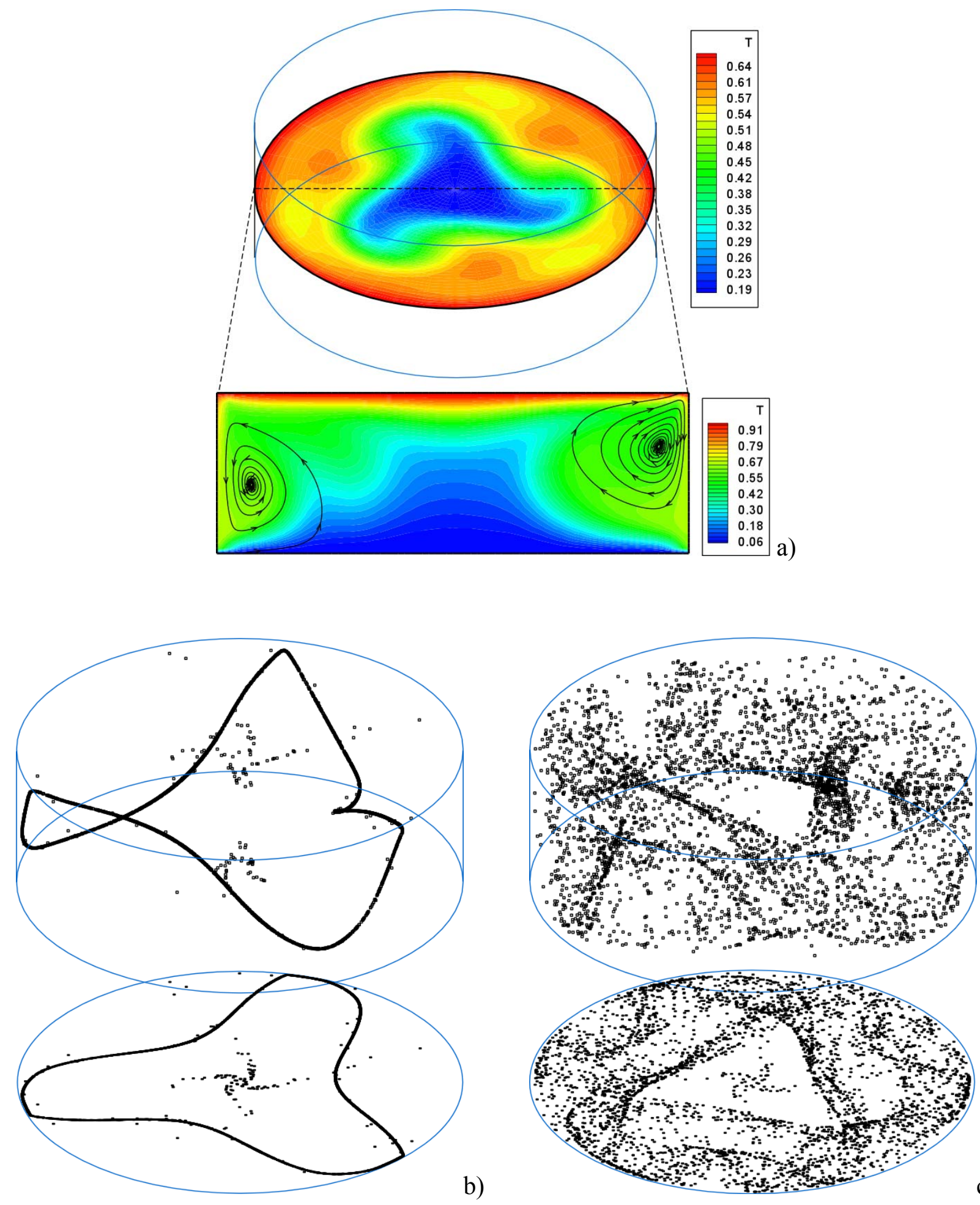

Figure 2: Snapshot (a) of traveling wave state (liquid bridge of $\mathrm{NaNO}_{3}, \mathrm{Pr}=8, \mathrm{~A}=0.34, \mathrm{Ma}=20600$ ) and $3 \mathrm{D}$ view of spatial particle distribution (and related projection in the xy plane) for two values of the St parameter: (b) $\xi=1.85$, St $\cong 10^{-4}$ (snapshot of PAS at $\mathrm{t}=\tau_{\mathrm{PAS}} \cong 2 \times 10^{-1}$ ) and (c) $\xi=1.85, \mathrm{St} \cong 10^{-5}$ at $\mathrm{t}=50 \mathrm{x} \tau_{\text {PAS }}$ where $\tau_{\text {PAS }} \cong 2 \times 10^{-1}$ is the nondimensional time required for PAS formation in (b) (in this case no one-dimensional PAS is formed, a similar behavior occurs for $\xi=1$ and $\mathrm{St} \cong 10^{-4}$ ). 
Results of the validation study are shown in Table I. Validation has been achieved by comparison with the numerical results of Melnikov et al. (2011).

Particles $\left(5 \times 10^{3}\right)$ were seeded uniformly into the computational domain assuming the initial velocity of each particle equal the local fluid velocity. The value of $\eta$ considered for this simulation was $\eta=1 \times 10^{-5}$, which corresponds to the particles with density (solid/fluid) ratio $\xi=1.85$ and size $45 \mu \mathrm{m}$ considered by Melnikov et al. (2011). Figure 2c illustrates the typical distribution of particles obtained for $\eta=1 \times 10^{-6}$, which indicates that no PAS structures are formed unless $\eta$ is larger than $a$ threshold value. This aspect is of crucial importance in demonstrating the reliability of the present algorithm (the interested reader is referred to Lappa, 2013a,b for additional details on the validation strategy and related underlying criteria). Here we limit ourselves to mentioning that we performed a simulation of particle motion by setting $\xi=1$ and $\mathrm{St}=10^{-4}$ and albeit the very long time simulated (50 times the time required for PAS formation when $\eta$ is in the right range) no stable or recognizable association of particles was observed. Rather, the emergence of PAS was obtained only when the size of tracers exceeded the same critical size (inertial parameter) identified by Melnikov et al. (2011) (numerical simulations) and Schwabe et al. (2007) (experiments), which is at the basis of the validation criterion used here.

\subsection{Scheme Order and Error Analysis}

Unlike earlier articles where the particle equation was solved "separately" (the 3D solution was frozen to save computational time and the particle tracking equation solved using such a frozen solution as a "background" state), here eq. (14) has been dynamically integrated together with equation (8-13) (i.e. with the same time integration step).

This is an important difference. Given the small value of the nondimensional time integration step $\Delta \mathrm{t}$ required for the stability of the Navier-Stokes algorithm $\left(\Delta \mathrm{t} \cong 5 \times 10^{-8}\right)$, one is not forced to use high-order schemes for the integration of eq. (14). Even with a first-order scheme, in fact, the error produced at each step (the so-called local truncation error) is $\mathrm{O}\left(\Delta \mathrm{t}^{2}\right)$, which accumulated over the number of iterations $\mathrm{N}_{\text {iter }}$ required for PAS formation in the case considered here (typically $\mathrm{N}_{\text {iter }} \cong 4 \times 10^{6}$ ), gives a global truncation error (Atkinson, 1989) $\mathrm{N}_{\mathrm{iter}} \Delta \mathrm{t}^{2}=\mathrm{O}\left(10^{-8}\right)$.

\section{Results}

For concreteness we focus on the same liquid bridge already used for the validation study (extensively investigated in earlier analyses).

By taking periodic accelerations into account, we now introduce a new degree of freedom into the problem. An obvious question that arises concerns the potential modification of the earlier findings by the inclusion of periodic vibrations.

Particularly interesting is the interplay between the transport of solid particles by Marangoni flow, on the one hand, and transport phenomena in fluid phase induced by gjitters accelerations, on the other hand. While it is well known that steady gravity levels may induce sedimentation of the freely 
suspended particles (see, e.g., Raju and Meiburg, 1995; Pasquero et al., 2003; Schwabe and Mizev, 2011), the role played by periodic accelerations in the dynamic process of PAS formation is still poorly understood. Potentially, they could jeopardize the outcome of experiments planned for this specific subject onboard the ISS.

The properties of the considered fluid system are summarized in Table II.

TABLE II: Fluid system properties $\left(\mathrm{NaNO}_{3}\right)$

\begin{tabular}{ll}
\hline \hline Fluid density $\rho, \mathrm{kg} \mathrm{m}^{-3}$ & 1904 \\
Kinematic viscosity $\mathrm{v}, \mathrm{m}^{2} \mathrm{~s}^{-1}$ & $1.27 \times 10^{-6}$ \\
Thermal diffusivity $\alpha, \mathrm{m}^{2} \mathrm{~s}^{-1}$ & $1.58 \times 10^{-7}$ \\
Surface tension temperature coefficient $\sigma_{\mathrm{T}}, \mathrm{N}(\mathrm{mK})^{-1}$ & $7 \times 10^{-5}$ \\
Thermal expansion coefficient $\beta_{\mathrm{T}}, \mathrm{K}^{-1}$ & $1.25 \times 10^{-3}$ \\
Liquid bridge Axial extension $\mathrm{L}, \mathrm{m}$ & $6.8 \times 10^{-3}$ \\
\hline \hline
\end{tabular}

Assuming as diameter of the liquid bridge $2 \times 10^{-2} \mathrm{~m}\left(\rightarrow \mathrm{L}=6.8 \times 10^{-3} \mathrm{~m}\right)$, the temperature difference corresponding to the considered value of the Marangoni number (20600) is $\Delta \mathrm{T} \cong 16.5 \mathrm{~K}$ (which gives $\beta=\beta_{\mathrm{T}} \Delta \mathrm{T} \cong 2.07 \times 10^{-2}$ ).

The density and size of the particles are assumed to be fixed $\left(\xi=1.85, \mathrm{St} \cong 10^{-4} \rightarrow \eta \cong 10^{-5}\right)$. The acceleration amplitude and angular frequency of the monochromatic disturbance vary in the ranges $10^{3} \leq \gamma \leq 10^{6}$ and $10 \leq \omega \leq 5 \times 10^{4}$, respectively (for each generic interval $10^{\mathrm{n}-1} \leq \gamma<10^{\mathrm{n}}$ and $10^{\mathrm{n}-1} \leq \varpi \leq 10^{\mathrm{n}}$, in particular, numerical simulations have been performed for $\gamma=\mathrm{c} 10^{\mathrm{n}-1}$ and $\varpi=\mathrm{d} 10^{\mathrm{n}-1}$ considering for both c and d values 1, 3, 5 and 10).

As anticipated in the introduction, the overall goal here is the derivation of a quantitative map (in the $\gamma-\varpi$ plane) for the occurrence of PAS with the express intent to support the optimization of a set of experiments to be performed in space. Beyond practical applications, it is rather clear that these problems also exert an appeal on researchers and scientists as a consequence of the complexity of the possible stages of evolution, of the non-linear behavior and because these organized particle structures are aesthetically and philosophically pleasing as well as irresistible to theoretical physicists (Balkovsky et al., 2001; Saw et al., 2008; Di Carlo et al., 2009). Moreover, the fact that strikingly well-ordered and similar phenomena are found also in other systems (not just physical, but also chemical and biological systems; see, e.g., Lappa et al., 2002; Carotenuto et al., 2002; Lappa, 2003b and 2011), indicates that these subjects really stand at the intersection of many scientific branches (which make them a multi-domain field of investigations and a truly interdisciplinary science).

\subsection{Gjitters perpendicular to the liquid bridge axis.}

A summary of the results in the case of gjitters directed along a direction perpendicular to the axis of the liquid bridge is shown in Fig. 3. In this map (a diagram with $\varpi$ as abscissa and $\gamma$ as ordinate) 
three distinct regions can be clearly discerned. The first one is the region of "standard" PAS formation, that is the area of the space of parameters $(\gamma, \varpi)$ where the PAS formation seems to be unaffected by the presence of vibrations (regardless of their acceleration amplitude and frequency). From a purely technical standpoint, this region would correspond to "safe" (ideal) conditions for the execution of a space experiment aimed at studying PAS emergence and related formation mechanism.

The opposite situation, i.e. the region in which no PAS occur, can be also clearly identified in Figure 3 (this should be regarded as the worst case as opposed to the ideal situation of standard PAS emergence).

Most interestingly, a third narrow region also exists, which separates the two limit situations discussed above (the transition boundaries of this area are indicated by means of solid lines in Fig. 3). As expected, in this region some interesting and exotic dynamics can be discerned and described (they are the main subject of the present section). These behaviors are driven by the competition between the two involved counteracting forces exerting their influence on the considered phenomena, these being: the "capturing" action exerted on solid particles by the attractive close manifold existing in the traveling wave state of supercritical Marangoni flow as discussed by Pushkin et al. (2011) and Lappa (2013a) (on which particles would tend to accumulate and order in the absence of vibrations), and the disturbing effect played on each particle by the inertial disturbances produced by vibration-induced accelerations (eq. 2 ).

Here, we introduce a general classification of the observed behaviors based on the degree of residual disorder and unsteadiness displayed by the recognizable pattern. In particular, the PAS variants that can be identified on the basis of this approach are referred to as asymmetric, fringed, hammered and jagged PAS.

By the first category we intend PAS for which a clear ordering of particles along a single closed line (one-dimensional circuit) can still be identified, but the pattern resulting from the projection of the $3 \mathrm{D}$ curve on the xy plane no longer exhibits the original symmetry with the set of identical lobes (petals) shown in Fig. 2 b (see Fig. 4a,b).

The second type of PAS consists of accumulation of particles for which some branches are still featured by the collapse of particles into a single (purely one-dimensional) portion of curve, whereas other parts display particles "fluctuating" in a given neighborhood of the ideal line which would be formed without vibrations (Fig. 4d).

The hammered PAS (Fig. 4e) is termed in this way because of the peculiar features of its structure, which seems to be given by a collection of "segments" of different inclination attached one another (forming a polygon-like circuit).

The last category is given by PAS with a more or less regular shape (no asymmetry or "segments" visible), for which, however, particles uniformly disperse over a region of finite (radial) thickness (Fig. 4h). Obviously, mixed "states" are also possible.

At this stage, a further characterization of the PAS map shown in Fig. 3 can be obtained by imagining to move in the $(\gamma-\varpi)$ plane along a line of constant $\gamma$ or constant $\varpi$ (horizontal or vertical 
traverse lines or traces, respectively), until the considered line intersects one of the (solid line) transition curves.

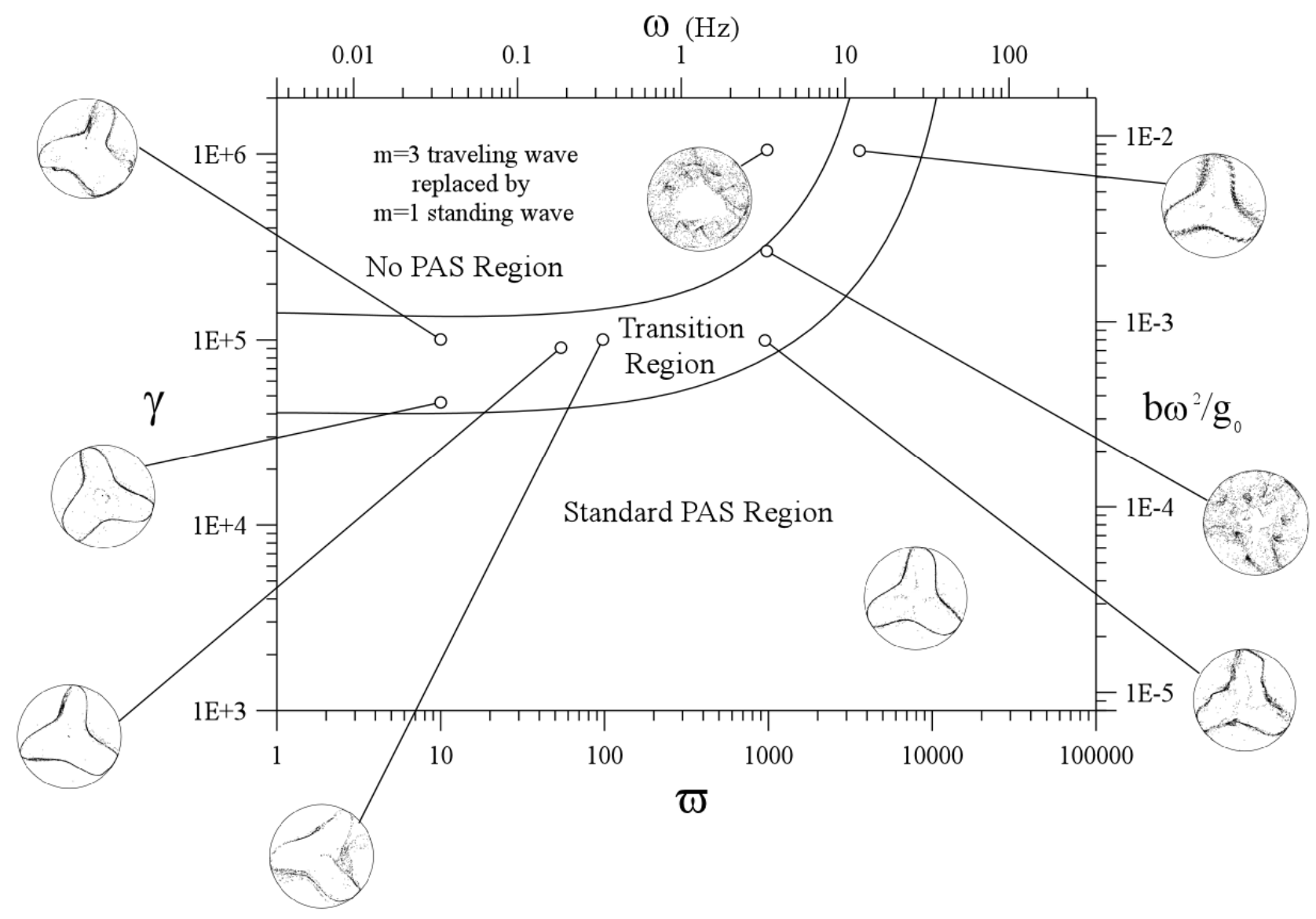

Figure 3: Map for the occurrence of PAS as a function of acceleration amplitude and frequency in the case of gjitters perpendicular to the axis of the liquid bridge (only a subset of performed simulations is shown).

Let us consider for instance a horizontal traverse at $\gamma=10^{5}$.

The pattern at $\varpi=10$ (a snapshot is shown at $4 \mathrm{~b}$ ) is a typical example of PAS pertaining to the first kind defined above. Its petals are no longer equally sized and spaced. In particular, a periodic disturbance affecting the size and shape of petals can be clearly identified (as witnessed by the fact that one petal grows in time while the other shrinks), with a percentage of variation in the azimuthal extension of each petal that can reach $70 \%$ of its ideal "unperturbed" (no gjitter) condition. 


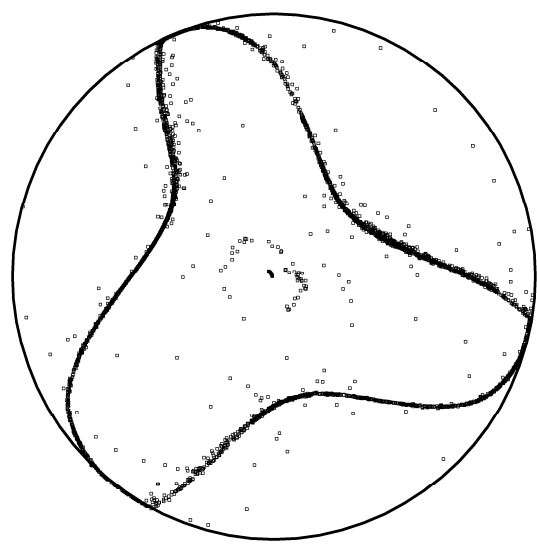

a)
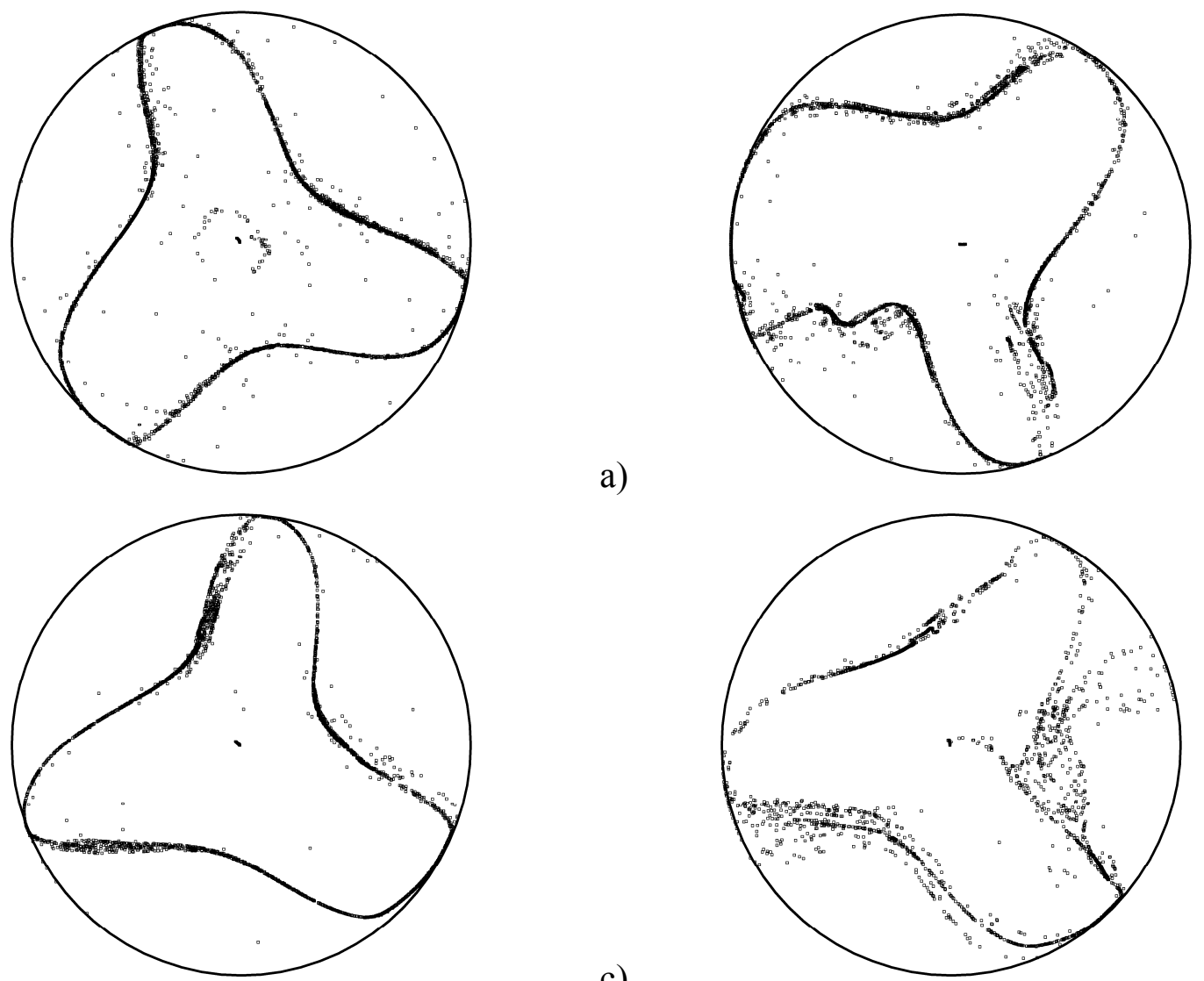

c)

d)
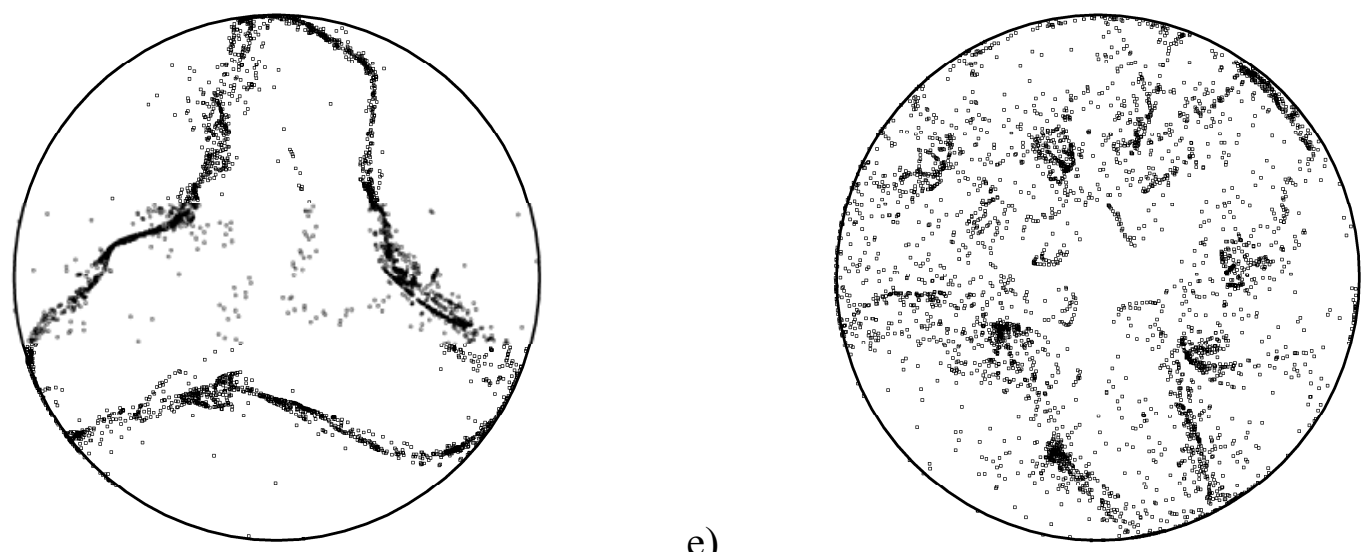

b)

e)
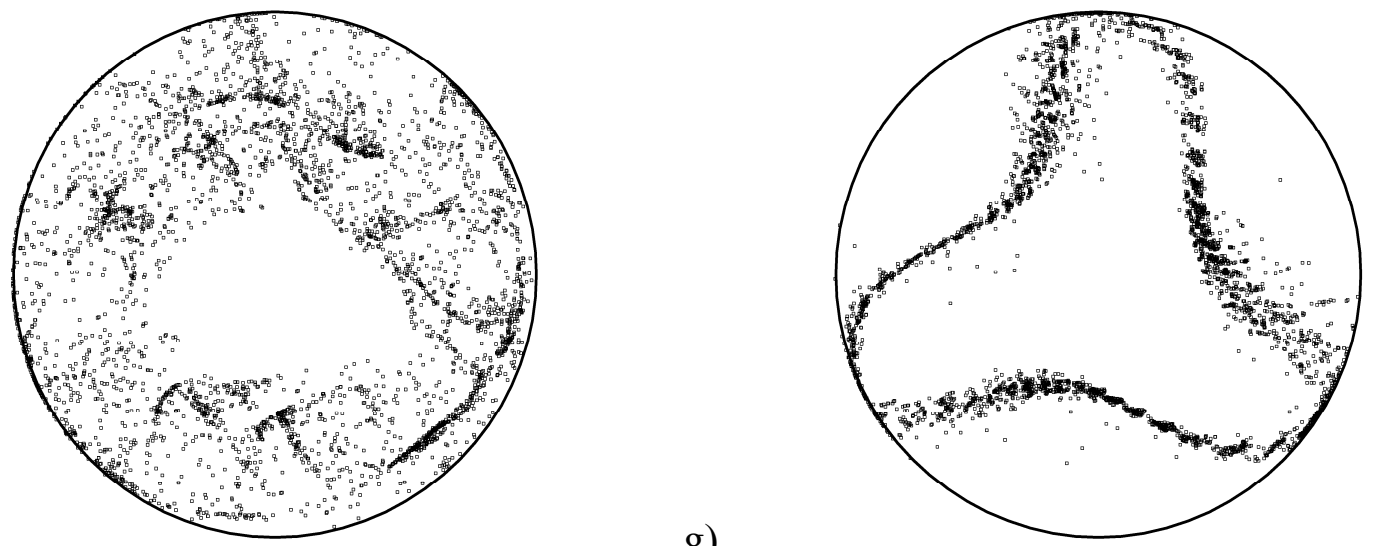

f)

g)

Figure 4: Snapshot of PAS for gjitters acting along a direction perpendicular to the liquid bridge axis $(\phi=0)$ and distinct values of acceleration amplitude and frequency: a) $\left.\gamma=5 \times 10^{4}, \varpi=10, b\right) \gamma=10^{5}$, $\varpi=10$, c) $\gamma=10^{5}, \varpi=70$, d) $\gamma=10^{5}, \varpi=10^{2}$, e) $\gamma=10^{5}, \varpi=10^{3}$, f) $\gamma=3 \times 10^{5}, \varpi=10^{3}$, g) $\gamma=10^{6}, \varpi=10^{3}$, h) $\gamma=10^{6}, \varpi=5 \times 10^{3}$. 
As shown in Fig. 4c, interestingly, an increase in $\varpi(\varpi=70)$, can cause a decrease in such a percentage (indeed, the size distribution of lobes and their shapes look more regular in Fig. 4c).

As $\varpi$ is further increased (see, e.g., Fig. $4 \mathrm{~d}$ for $\varpi=10^{2}$ ), however, the progressive reduction of such a large-scale disturbance is taken over by the emergence of a new class of disturbances appearing on a smaller scale. They consist of a cyclic departure of particles from the purely one-dimensional path that would be maintained in the case of no gjitters (many tracers are observed to detach periodically from the thread structure giving the impression of swarms of "rebel" particles fluctuating in a limited neighborhood of the ideal one-dimensional PAS). This behavior is typical of "fringed" PAS. At progressively higher gjitters frequencies $\left(\varpi \geq \mathrm{O}\left(10^{3}\right)\right)$, the large-scale disturbance disappears completely while the amplitude of the smaller-scale disturbances shrinks (by disturbance "amplitude" here we mean the radial excursion of the rebel particles). This results in a more regular pattern, which, however for $\varpi=10^{3}$ (Fig. 4e), still displays a departure from standard PAS in the form of localized particle "explosions" (radial tracer displacements concentrated in some specific points). This peculiar behavior leads to the illusions of a closed line formed by the conjunction of distinct parts of different size and inclination (the hammered PAS). For $\varpi>2 \times 10^{3}$, one recovers the standard (defect-less) PAS.

A description of the system behavior at fixed values of $\varpi$ (vertical traverses) is also instructive.

For $\varpi=10^{3}$, as an example, the system evolves from the standard PAS at $\gamma=10^{4}$, to no onedimensional PAS at $\gamma=10^{6}$ (Fig. 4g), passing through the hammered PAS that we have seen before for $\gamma=10^{5}$, and a more turbulent hammered PAS at $\gamma=3 \times 10^{5}$ for which the particle "explosions" become more evident and destructive (Fig. 4f).

As a further example, for $\varpi=3 \times 10^{3}$, increasing the gjitter amplitude from $\gamma=10^{5}$ to $\gamma=10^{6}$, causes the transition from the standard PAS to the "jagged" PAS (shown in Fig.4h); this last PAS type can be distinguished from the hammered variants in virtue of its regular shape and the peculiar property of being constituted by the ordered association of "clots" of particles. Finally, if $\varpi$ is increased to $10^{4}$ also this pattern disappears (with recovery of the standard PAS).

In practice, Figure 3 shows that in the range of acceleration amplitudes considered $\left(\gamma \leq 10^{6}\right)$ and $\varpi=10^{4}$, the standard PAS is attained regardless of the specific value of $\gamma$.

Let us recall at this stage that, even though, as just stated above, gjitters seem to have no effect in the high-frequency part of the spectrum, the results summarized in Figs. 3 and 4 have been obtained without consideration of the Basset force. As discussed in Sect. 2.4, this force is expected to play some role in the region of the plot with $\varpi>5 \times 10^{3}$, which calls for a proper justification of its omission in the governing particle-tracking equation.

As in the present study the maximum considered frequency has been fixed to $\varpi=5 \times 10^{4}$, this separate analysis has been expressly concentrated on values $\mathrm{O}\left(10^{4}\right)$ of the nondimensional frequency. Moreover, given the extremely time-consuming and memory-consuming nature of the algorithm required for the computation of the history term (one is forced to store in the computer memory the overall "history" of the $\underline{V}_{\text {part }}-\underline{V}$ quantity), the simulation has been limited to considering just one (single) particle (whose trajectory in the rotating reference frame has been determined for both cases of Basset term included and neglected). 
Related results are shown in Fig. 5.
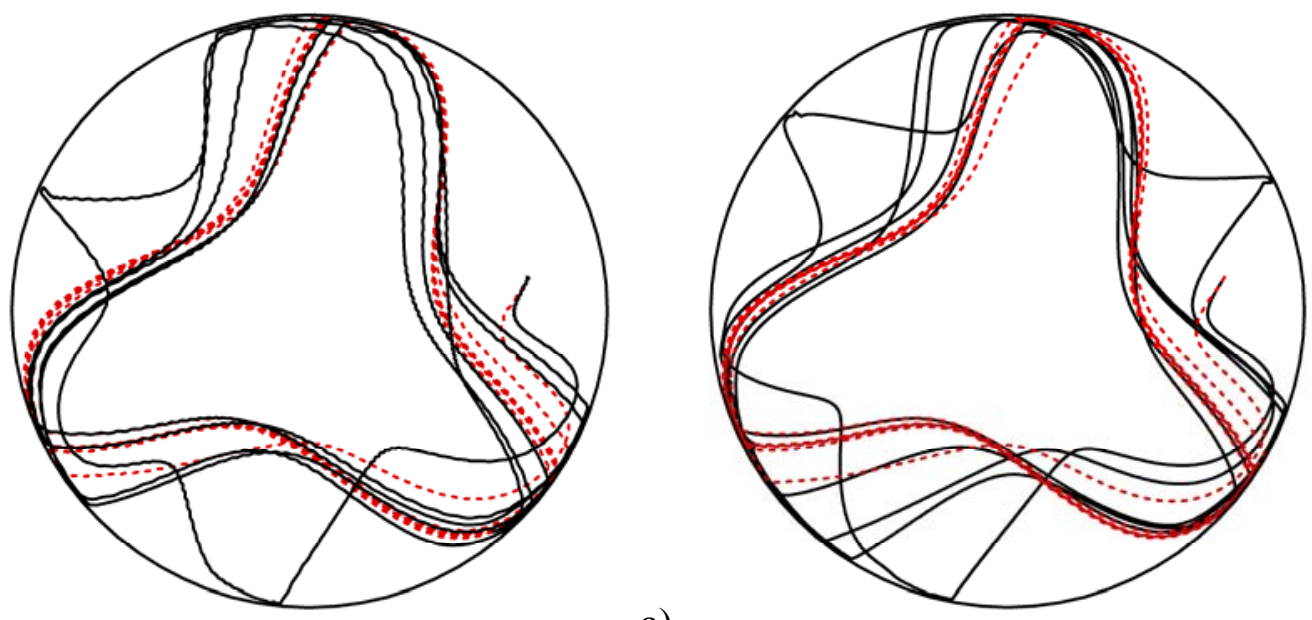

a)

b)

Figure 5: Trajectory in the rotating frame of reference of a particle with initial position $\mathrm{r}=0.77 \mathrm{R} / \mathrm{L}$, $\mathrm{z}=0.2 \varphi=0$, with (solid line) and without (dashed line) the Basset force: a) $\left.\gamma=10^{6}, \varpi=10^{4} ; \mathrm{b}\right) \gamma=10^{6}$, $\varpi=5 \times 10^{4}$.

Albeit some differences can be noted in the initial stage of evolution, the plot shows that both trajectories (with and without Basset force) collapse after some time on the same one-dimensional pattern (that corresponds to the typical defect-less shape of PAS, by which it can be concluded that for the considered conditions the influence of the history term on the asymptotic dynamics of PAS can be assumed to be negligible when $\left.\varpi=\mathrm{O}\left(10^{4}\right)\right)$.

\subsection{Gjitters parallel to the liquid bridge axis.}

Now we embark in the description of results for the case in which the angle formed by gjitters and the equatorial plane is $\phi=90^{\circ}$.

A direct comparison of Fig. 6 with Fig. 3 leads to the more or less immediate realization that gjitters parallel to the axis of the liquid bridges are, in general, less "dangerous" than those acting along directions contained in an equatorial plane. This is clearly witnessed by the position and shape of the curve bounding the no-PAS region, which is shifted to higher values of $\gamma$, hence, giving rise to a smaller no-PAS area and a broader transition zone.

Many of the PAS categories introduced in Sect. 3.1 according to the spatial scale of disturbances affecting the PAS shape and particle distribution are still applicable to the present case. Indeed, examples of patterns pertaining to the hammered and jagged categories can be clearly seen in Fig. 7e and 7h, respectively. Interestingly, Figure 7, however, also shows some additional varieties in terms of PAS characteristics which are not present in the $\phi=0^{\circ}$ case; we refer, in particular, to Figs. $7 \mathrm{a}-\mathrm{b}$ and $7 \mathrm{c}-\mathrm{d}$, which lead to the definition of two new categories as further described below: The first class (Fig. 7a-b) is hereafter referred to as D-PAS (where the letter D denotes "double") owing to the typical topology displayed by the resulting pattern, which is no longer featured by a 
single loop, showing rather the coexistence of two distinct loops separated by an average radial distance dependent on the specific gjitters amplitude $\gamma$ considered.

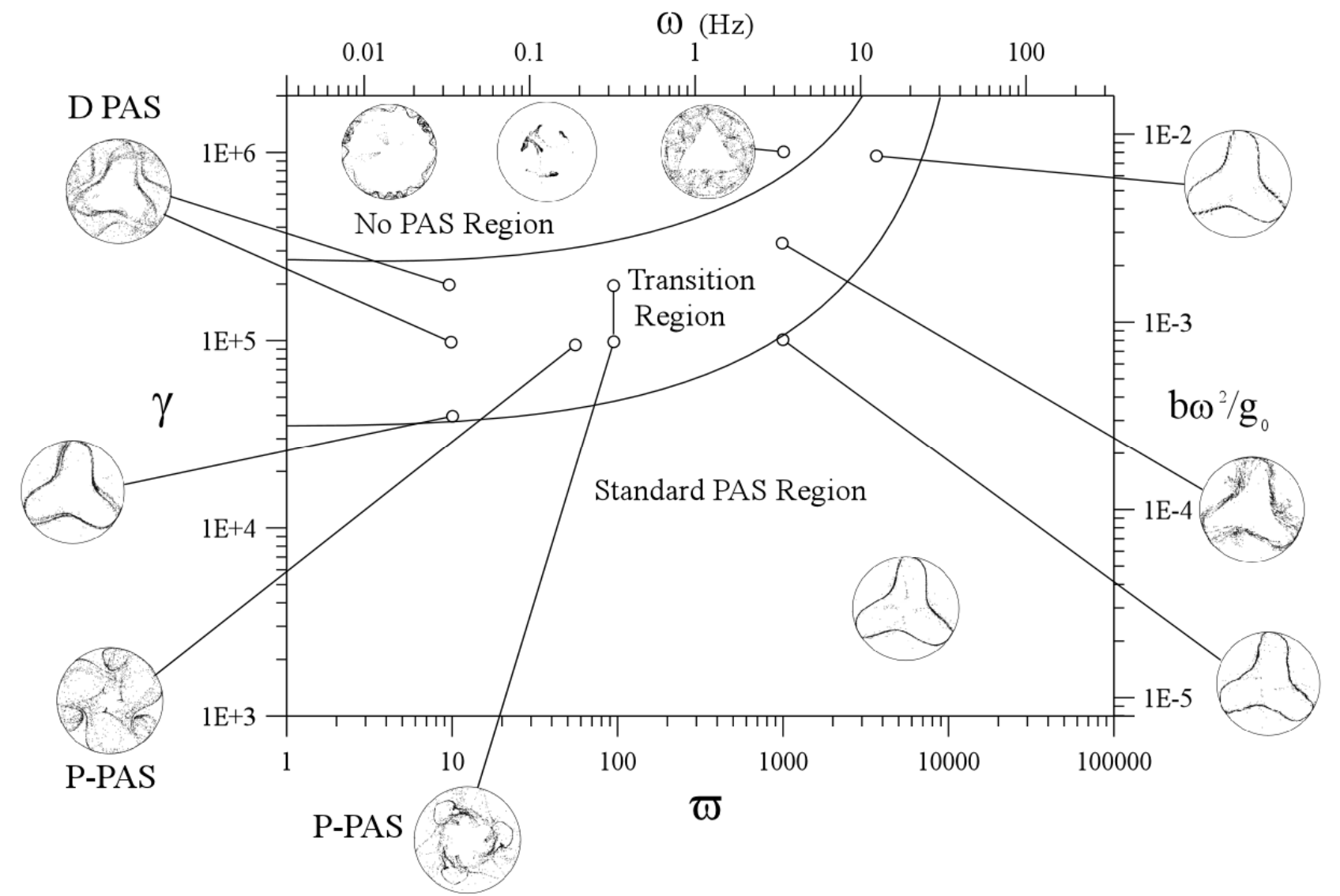

Figure 6: Map for the occurrence of PAS as a function of acceleration amplitude and frequency in the case of gjitters parallel to the axis of the liquid bridge (only a subset of performed simulations is shown).

This new category can be met moving along the vertical traverse at $\varpi=10$. The standard PAS existing at $\gamma=10^{4}$ is replaced by the D-PAS at $\gamma=3 \times 10^{4}$ (see, e.g., Fig. 7 a for $\gamma=5 \times 10^{4}$ ) for which the two loops are very close each other; a further increase of $\gamma$, however, causes a significant increase of the average radial distance between them (see Fig. $7 \mathrm{~b}$ for $\gamma=10^{5}$ ), until no recognizable PAS can be obtained at $\gamma=5 \times 10^{5}$.

This pattern is not entirely new as it has been often observed in the framework of ground-based experiments (see, e.g., Ueno et al., 2000). This is not surprising if one considers that for this frequency, the inertial disturbance may be seen as a quasi-steady residual $\mathrm{g}$ (for the considered $\mathrm{NaNO}_{3}$ liquid bridge with height $6.8 \times 10^{-3} \mathrm{~m}, \varpi=10$ would correspond to a dimensional angular frequency $\omega=3.4 \times 10^{-2} \mathrm{~Hz}$ ). Such analogy may be seen as a hint for a possible role played by steady gravity levels in determining the splitting of the original PAS single-loop circuit in two loops. Ideas along these lines are not new and can be found, for instance, in Schwabe et al (2007) and Schwabe and Mizev (2011), where gravity was expressly indicated as the main cause for heavy $(\xi>1)$ particles leaving the fluid trajectories and ensuing broadening of the observable part of the PAS (it is a well-known fact that gravity can even prevent PAS formation in some circumstances and/or suppress the Marangoni flow instability, Lappa et al., 2000). 

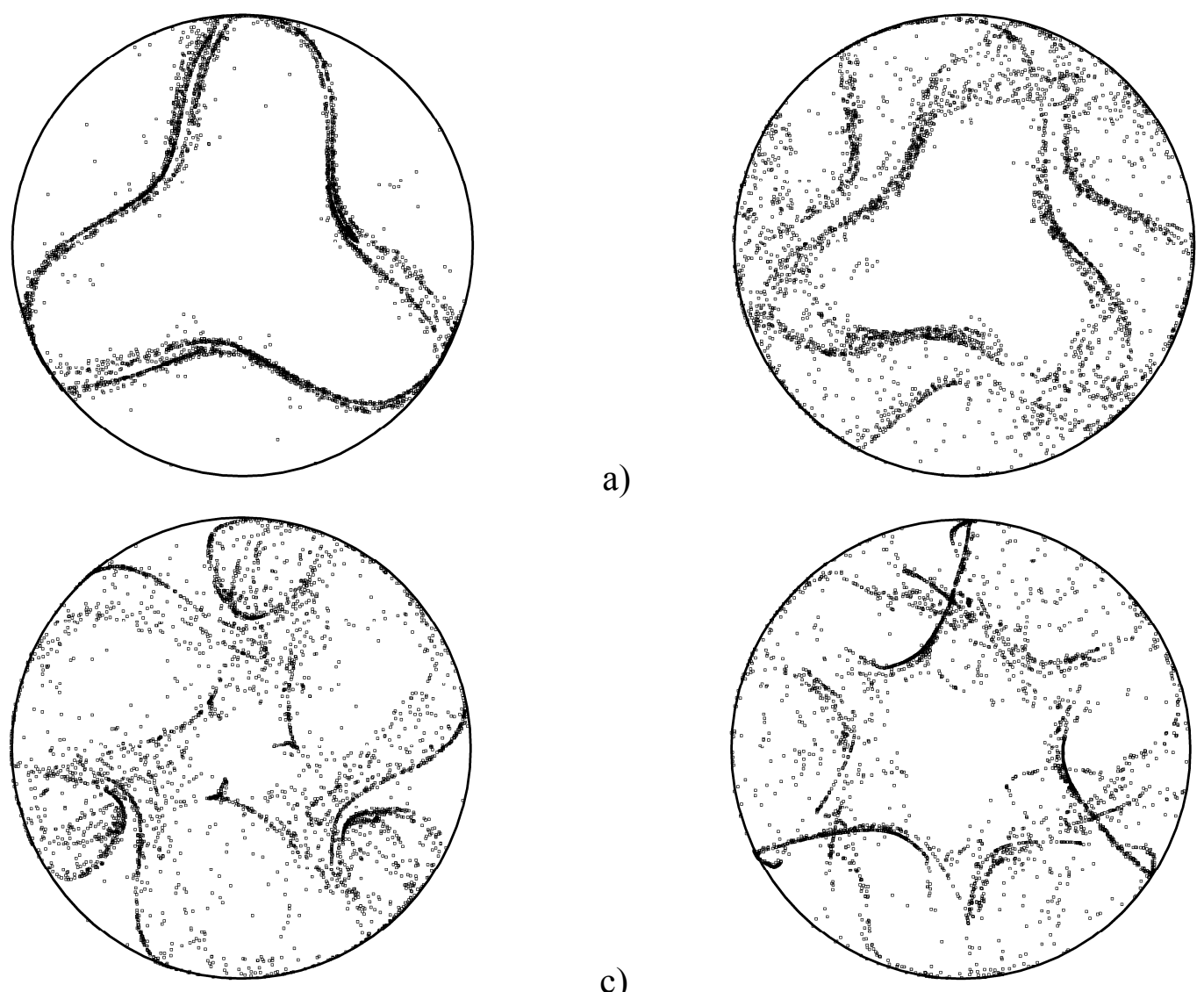

a)

b)
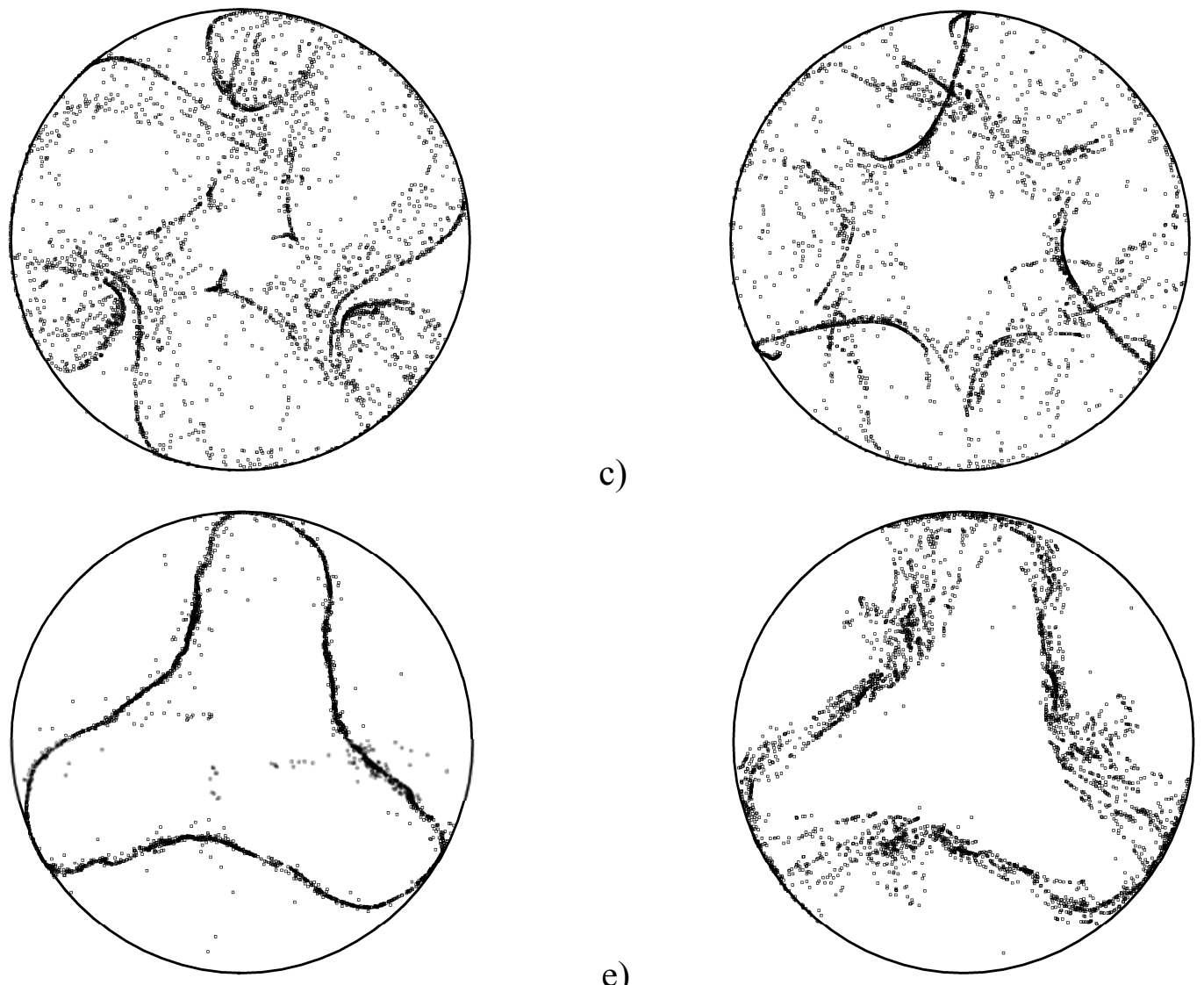

c)

d)
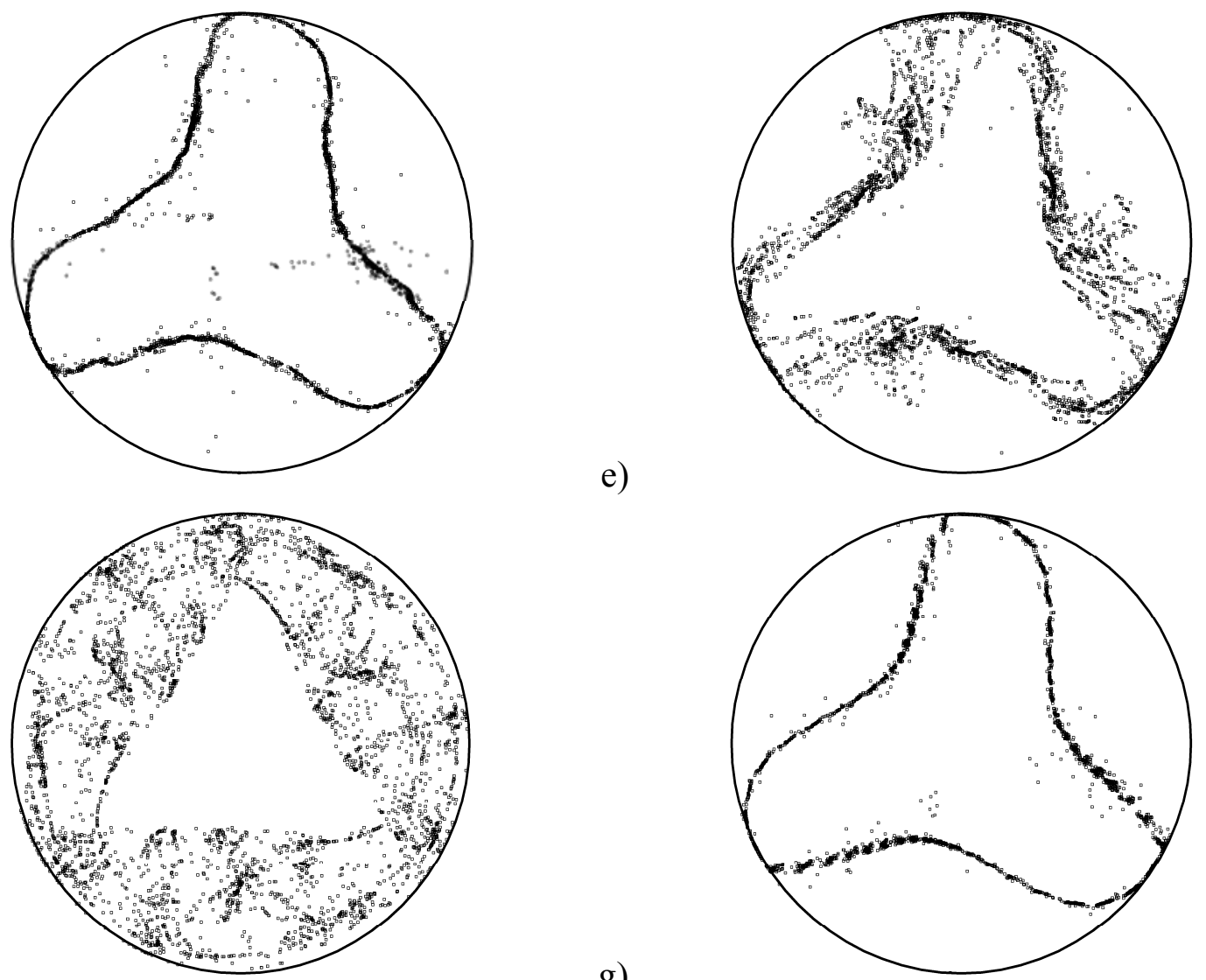

e)

g)

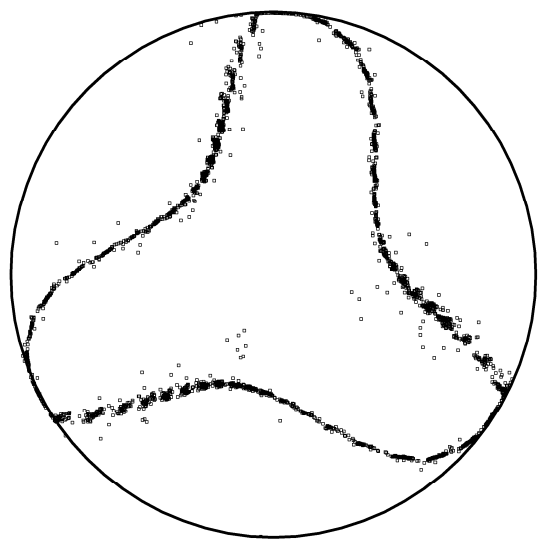

h)

Figure 7: Snapshot of PAS for gjitters acting along the liquid bridge axis $(\phi=\pi / 2)$ and distinct values of acceleration amplitude and frequency: a) $\left.\gamma=5 \times 10^{4}, \varpi=10, b\right) \gamma=10^{5}, \varpi=10$, c) $\gamma=10^{5}, \varpi=70$, d) $\gamma=10^{5}, \varpi=10^{2}$, e) $\gamma=10^{5}, \varpi=10^{3}$, f) $\gamma=3 \times 10^{5}, \varpi=10^{3}$, g) $\gamma=10^{6}, \varpi=10^{3}$, h) $\gamma=10^{6}, \varpi=5 \times 10^{3}$. 


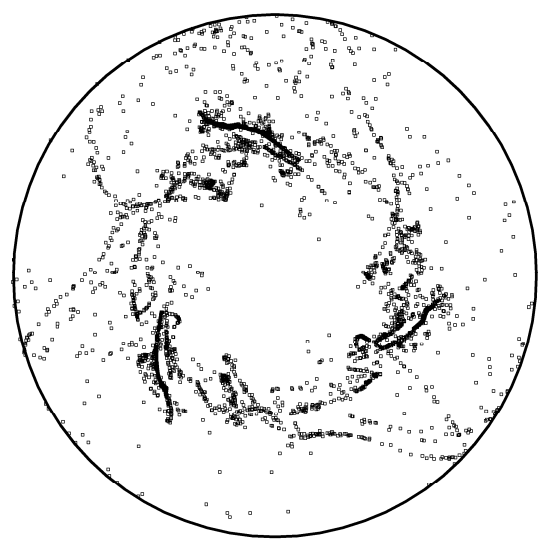

a)
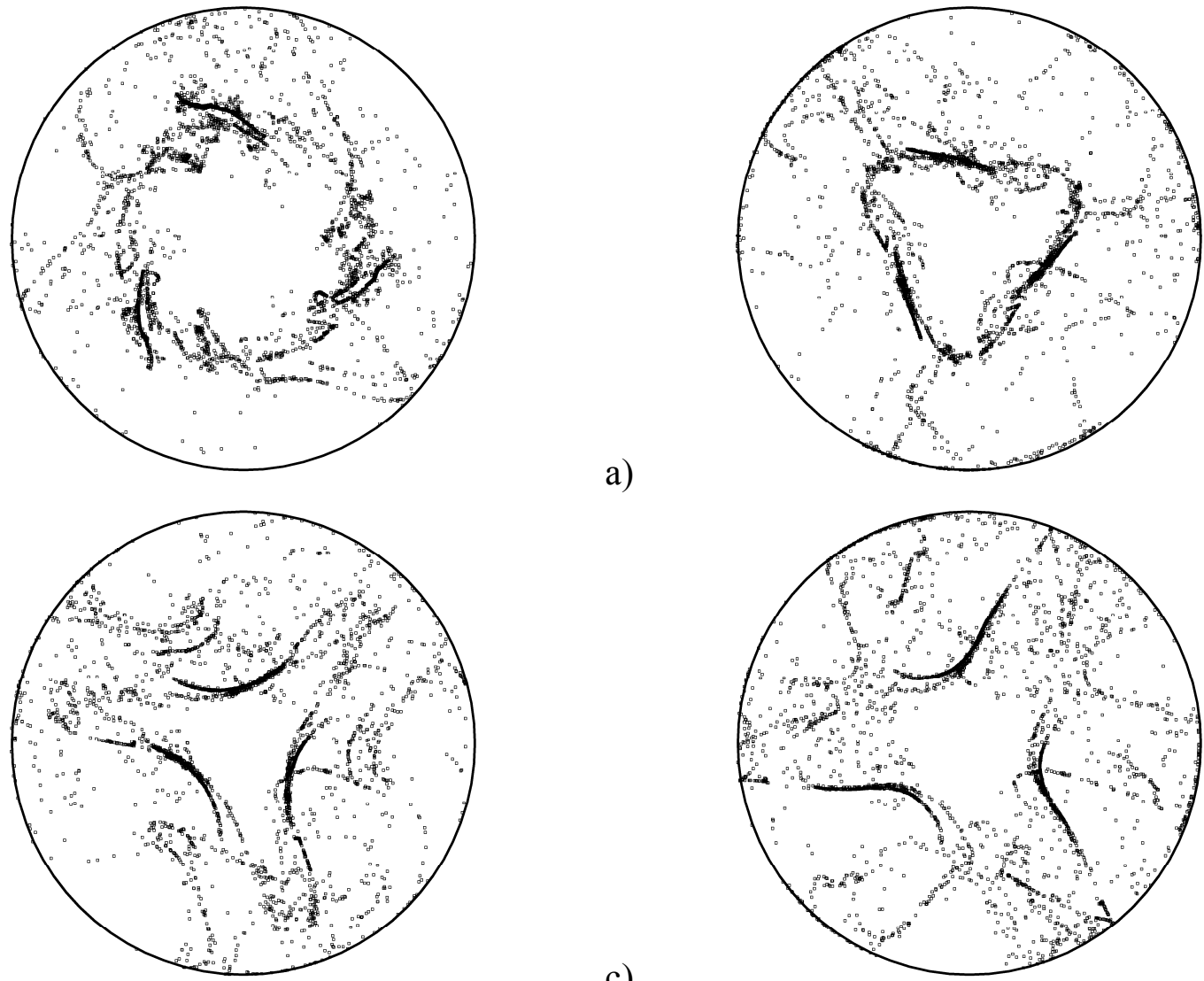

b)

c)

d)
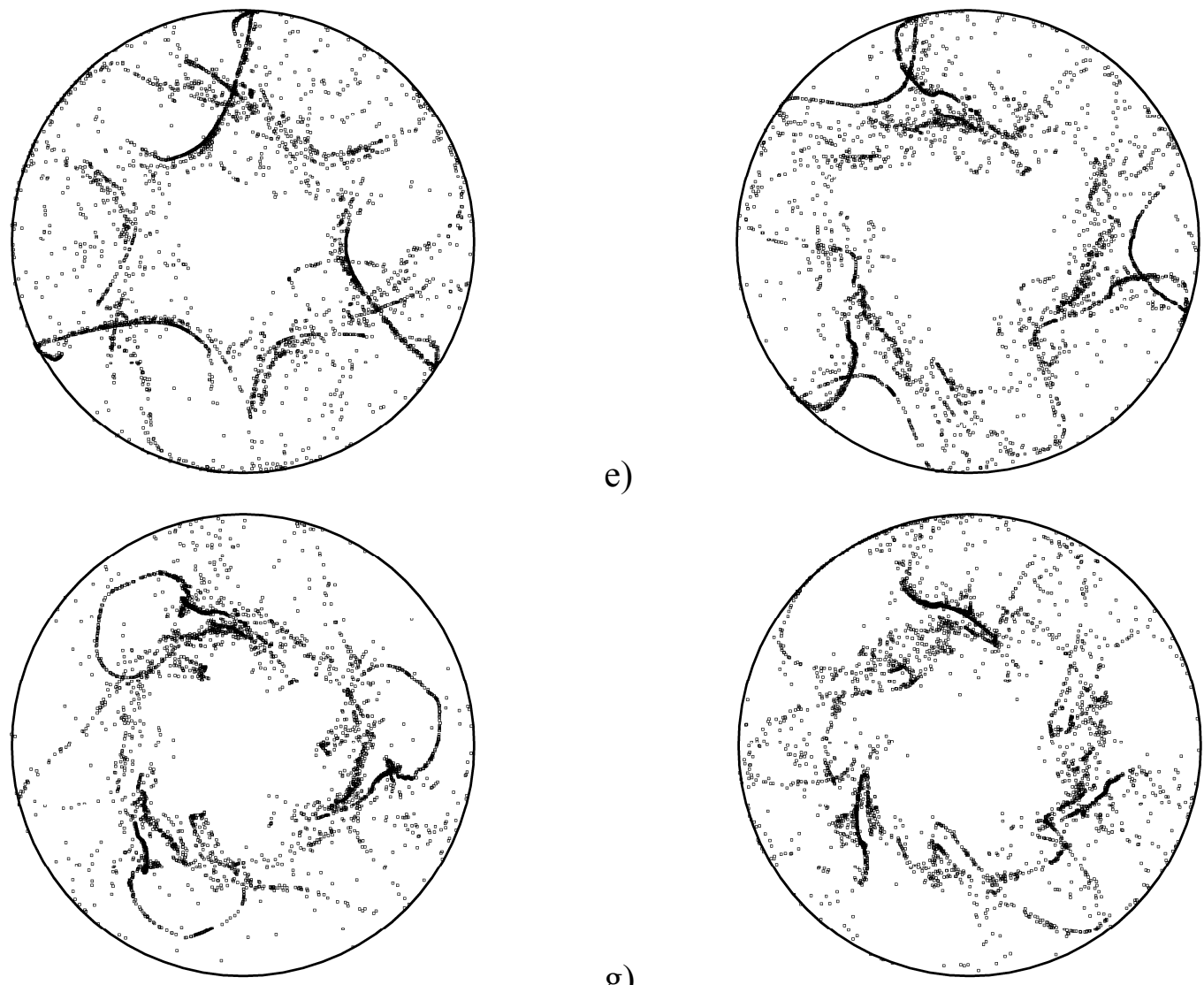

e)

g)

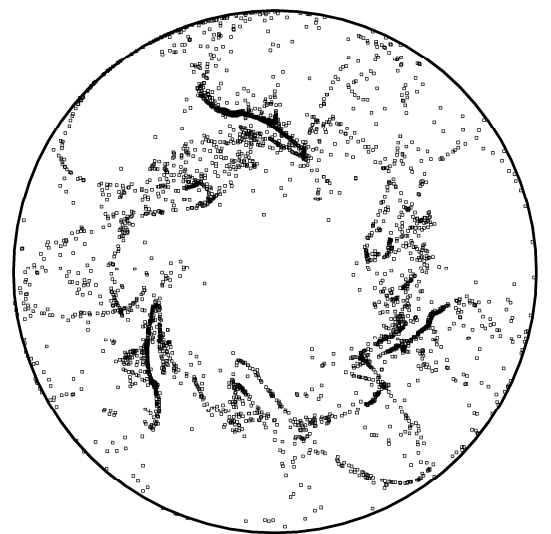

h)

Figure 8: Oscillatory Pulso-rotating PAS for $\phi=\pi / 2, \gamma=10^{5}, \varpi=10^{2}$ : particle distribution as seen from above in the laboratory reference frame is shown in eight snapshots evenly distributed during one period of oscillation $\tau \cong 6.5 \times 10^{-2}$. 
The second new category is even more interesting in terns of patterning characteristics and spatiotemporal behavior. Concentrating on the horizontal traverse at $\gamma=10^{5}$, examples of this kind of PAS can be seen for $\varpi=70$ and 100 (snapshots are given in Figs. 7c and d).

The most remarkable property of this kind of phenomena is the strong time-dependent nature of the structures formed by the accumulation of solid particles (P-PAS), which give rise during one period of gjitter oscillation to a myriad of recognizable geometric shapes. Such geometric items (see Fig. 8) range from multi-armed windmills (Fig. 8a) to closed (Fig. 8b) or open triangles (Fig. 8c-d), to multi-sided star-shaped polygons (Fig. 8e), to fractal-like shapes (Fig. 8g shows three small triangle-like closed curves encapsulated in a larger-size triangle-like circuit), all alternating during one period of oscillation so as to create the illusion of a "pulsating" pattern in the reference frame rotating with the hydrothermal wave.

These specific dynamics may be seen as a consequence of the increased ability of inertial disturbances to interfere with the delicate mechanisms (Pushkin et al., 2011, Lappa, 2013a,b) driving PAS formation when the angular frequency of the inertial perturbation is comparable to the angular frequency of the hydrothermal wave, i.e. when these two quantities have the same order of magnitude $\mathrm{O}(\varpi)=\mathrm{O}(\Omega)$.

Indeed, an increase in $\varpi$ at constant $\gamma=10^{5}$ beyond $\varpi=3 \times 10^{2}$, determines a suppression of this fascinating pulsating behavior, with recovery at $\varpi=10^{3}$ of a stationary and fairly regular pattern as seen in the rotating reference frame (the weakly hammered PAS in Fig. 7e). This regularity, however, soon gives way to progressively less regular PAS as $\gamma$ is increased at constant $\varpi=10^{3}$. Beyond $\gamma=10^{5}$, the pattern becomes quite irregular and time-dependent (as seen in the rotating frame of reference); examples of this trend are shown in Fig. $7 \mathrm{f}$ for $\gamma=3 \times 10^{5}$ (that looks like a fringed PAS or a pattern with the characteristics of both hammered and jagged PAS) and Fig. $7 \mathrm{~g}$ for $\gamma=10^{6}$.

\section{Discussion}

\subsection{The effect of vibrations on particle motion}

Although the knowledge of the motion of solid particles in sinusoidally-vibrated systems seems to be still rather limited, some useful efforts can be found in the existing literature for the case of isothermal systems and relatively simple velocity fields. As an example, interesting studies of particle dynamics under the effects of small sinusoidal fluctuations in the free-stream velocity (i.e. harmonic background fluid velocity) are due to Lighthill (1954), Houghton (1961), Herringe (1977), Mei et al. (1991), Vojir and Michaelides (1994) and Coimbra and Rangel (2001). Other important investigations have dealt with the terminal velocity of a spherical particle in a vertically oscillating liquid (among them, it is worth mentioning Baird et al., 1967; Tunstall and Houghton, 1968 and Ikeda, 1989). For the case of particles undergoing a sinusoidal motion or subjected to vibrations in an otherwise quiescent fluid, we may cite Langbein (1991), Sun et al. (1994), Ellison et al. (1995), Thomson et al. (1997), Simic-Stefani et al. (2006), Hassan et al. (2006a), Hassan and Kawaji (2008). From all these works, it is known that, in general, a distinction must be introduced between 
the ranges of high and low frequency (the expected effects of gjitters being markedly different according to the range considered).

This was clearly assessed in the framework of space experiments (see, e.g., Sun et al., 1994 and Ellison, 1995), where it was directly observed that the effects of high-frequency gjitters are rather "localized" while the predominant gjitter-induced particle drifting movement results essentially from low frequency excitation.

From a purely theoretical standpoint, a general modus operandi in treating these subjects consists of splitting the particle velocity in two contributions, an average value $\underline{\bar{V}}_{\text {part }}$ plus a periodic oscillation $\underline{V}_{\text {part }}^{\prime}$. Defining the average contribution (in dimensional form) as:

$\bar{V}_{\text {part }}=\frac{\omega}{2 \pi} \int_{0}^{2 \pi / \omega} \underline{V}_{\text {part }} d t$

in turn, the time-dependent part $\underline{V}_{\text {part }}^{\prime}$ can be defined as:

$\underline{V}_{\text {part }}^{\prime}=\underline{V}_{\text {part }}-\underline{\bar{V}}_{\text {part }}$

In general, the amplitude of $\underline{V}_{\text {part }}^{\prime}$ can be represented with a Fourier expansion based on the fundamental gjitter frequency $\omega$ and its multiples:

$\left|\underline{V}_{\text {part }}^{\prime}\right|=\sum_{n=1}^{\infty} A_{n} \sin (n \omega t)$

In the majority of practical situations, however, the most significant contribution is given by the first term of the series expansion, i.e. the contribution oscillating at the same frequency of the gjitters:

$\left|\underline{V}_{\text {part }}^{\prime}\right| \cong A_{0} \sin (\omega t)$

Precise scaling arguments covering these quantities were elaborated even prior to the execution of dedicated space experiments. As an example, Langbein (1991) found for dilute dispersions of spheres the amplitude of the oscillatory part of particle velocity $\left(\mathrm{A}_{0}\right)$ to scale as $\gamma\left(\omega \widetilde{R}^{2} / 2 v\right)^{-1}$ in the range of high frequencies. In such a context it is also worth considering the excellent study by Simic-Stefani et al. (2006), who derived an analytic solution for the case of an isolated (single) particle. This analytic solution gives for $\varpi>\mathrm{O}\left(10^{3}\right)$ the same scaling relationship determined previously by Langbein (1991), whereas $\mathrm{A}_{0}$ is predicted to be directly proportional to $\gamma$ and almost independent of $\varpi$ for $\varpi<\mathrm{O}\left(10^{3}\right)$. 
These findings may be used to justify (at a minimum, they provide a reasonable hint along this line) the observed evolution of PAS and associated gjitters-induced defects when moving in the plane $(\gamma-$ $\varpi)$ along a horizontal traverse at constant $\gamma$ (or at constant $\varpi$ ).

We have seen in Sect. 3.1 and 3.2 that in the transition region the pattern displays (in the rotating reference frame where PAS should look stationary and behaving as an apparently solid unit in the absence of vibrations) a degree of "unsteadiness" whose scale depends (in the most general situation) on $\gamma$ and $\varpi$. In particular, when moving along a horizontal traverse at constant $\gamma$ (in the following we focus on $\left.\gamma=10^{5}\right)$ the pattern develops initially $\left(\varpi \leq \mathrm{O}\left(10^{2}\right)\right)$ large-scale deformations that are taken over by smaller scale turbulence as $\varpi$ further increases $\left(\varpi>\mathrm{O}\left(10^{2}\right)\right)$ (until a stationary PAS is recovered above a certain threshold $\varpi_{\text {ths }}$, which, as shown in Figs. 3 and 6, depends on the considered value of $\gamma$ ).

At this stage, in the light of the aforementioned scaling laws, the transition from large scale to small scale disturbances (and finally to a stationary solution) displayed by the particle pattern may be seen as the consequence of a decrease in amplitude of the oscillatory part of particle velocity as predicted by the relationship $\mathrm{A}_{0} \propto\left(\omega \widetilde{R}^{2} / 2 v\right)^{-1}$ (indeed, this may explain the observed evolution from fringed PAS (for $\phi=0$ ) and from P-PAS (for $\phi=90^{\circ}$ ) for $\varpi=\mathrm{O}\left(10^{2}\right.$ ) to the more regular hammered PAS for $\varpi=\mathrm{O}\left(10^{3}\right)$ and finally to stationary standard PAS for $\left.\varpi>\varpi_{\text {ths }}\right)$.

Similarly, the increase in the amplitude of both small and large scale defects, observed over the entire range of frequencies when $\gamma$ is increased at a constant value of $\varpi$, may be justified taking into account that, regardless of the specific value of $\varpi$ considered, $A_{0}$ is expected to be directly proportional to $\gamma$ (Simic-Stefani et al., 2006).

Albeit these arguments may appear convincing (at least in providing a qualitative interpretation of the observed dynamics), however, they are not exhaustive.

One must keep in mind, in fact, that, as shown by eq. (9) the effect of gjitters is not limited to the inertial action they exert on particles (Lappa and Carotenuto, 2003). In the presence of thermal or solutal gradients they can, in principle, produce their own convective fluid flow (e.g., Gershuni et al., 1994; Lizée and Alexander, 1997; Hirata et al., 2001; Dyko and Vafai, 2007; Srinivasan and Saghir, 2011) and influence in some circumstances other kinds of natural flows (of surface-tensiondriven nature in the present case) both in terms of strength and patterning behavior.

\subsection{Convective Flow of Vibrational Origin}

There is a long tradition of studies on this subject (the interested reader being referred, e.g., to Lappa, 2010 for a review).

In general, as explained before, we may expect gitters to have a two-fold effect on a non-isothermal dispersed multiphase flow (and related particle dynamics), the first being given by the action they exert directly on particle motion (with oscillatory part of the particle velocity obeying the scaling laws discussed before), the second coming from their influence on Marangoni convection via the so-called thermovibrational mechanism. 
A satisfactory treatment of the thermovibrational mechanism is beyond the scope of the present section (the interested reader being referred to, e.g., Savino and Lappa, 2003 and references therein). Here we limit ourselves just to highlighting that the specific interval of frequencies considered plays a critical role in determining the effect of gjitter on convective flow just as in the case of particle dynamics discussed before (see, e.g., Yan et al., 2005 and 2007; Dyko and Vafai, 2007; Ahadi and Saghir, 2012; Parsa and Saghir, 2012).

Like the velocity of a solid particle, the convective velocity field $\underline{V}_{\text {vibrat }}$ induced by a periodic acceleration in a generic point of the physical space can be seen as the sum of an average value $\underline{\bar{V}}$ plus a periodic oscillation of amplitude $\underline{V}^{\prime}$ (oscillating at the acceleration frequency $\omega$ and/or at frequencies that are multiples of $\omega$ ). As a result of such a convective field, the scalar quantities (temperature) are also distorted. These distortions in turn are also made up by a steady plus an oscillatory contribution.

The relative importance of the steady (average) and oscillatory contributions depends on the gjitter frequency. In particular, it is known that increasing the frequency from zero there is an initial regime characterized by a relatively large induced oscillatory velocity and oscillatory temperature with relatively small induced time-average contributions, whereas at high frequencies the steadyaveraged contributions become dominant. The latter case, in particular, can be treated within the framework of the so-called Gershuni's theory, which, from a purely mathematical standpoint, is valid in the limit of high-frequency $(\varpi>>1)$ and a small value $(<<1)$ of the parameter $\Lambda$ defined as:

$$
\Lambda=b \frac{\beta_{T} \Delta T}{L}=\frac{\beta \gamma}{\varpi^{2}}
$$

Several lines of evidence (originating from the original studies by Gershuni and Zhukhovitskii, 1981, 1986), in fact, have led to the realization that when such conditions are satisfied, the average flow induced by gjitters can be characterized/quantified more or less immediately in terms of a single parameter:

$$
R a_{V}=\frac{\left(b \omega \beta_{T} \Delta T L\right)^{2}}{2 v \alpha}=\frac{\left(\beta_{T} \Delta T L\right)^{2}}{2 v \alpha}\left(\frac{g_{\omega}}{\omega}\right)^{2}=\frac{\varpi^{2} \Lambda^{2}}{2 \operatorname{Pr}}=\frac{1}{2 \operatorname{Pr}}\left(\frac{\beta \gamma}{\varpi}\right)^{2}
$$

generally referred to as the vibrational Rayleigh number.

An effective assessment of the ranges (in terms of $\varpi$ ) of applicability of this theory for $\Lambda<<1$ and a value of the Prandtl comparable to that considered in the present work $(\operatorname{Pr}=\mathrm{O}(10))$ is due to Savino and Lappa (2003). In their work the Gershuni's simplified equations (and related implications) were shown to be valid for $\varpi>\mathrm{O}\left(10^{3}\right)$.

For the conditions considered here $\left(\gamma \leq 10^{6}\right)$, in particular, an evaluation of the $\Lambda$ parameter gives $\Lambda \leq 10^{-2}$ for $\varpi \geq 10^{3}$, which indicates that the vibrational Rayleigh number may be effectively used as a relevant parameter to characterize the flow in the high-frequency part of the spectrum $\left(\varpi>0\left(10^{3}\right)\right)$. 
Since this parameter takes for these conditions a very small value $\left(\operatorname{Ra}_{\mathrm{v}}<10\right.$ for $\gamma \leq 10^{6}$ and $\left.\varpi \geq 10^{3}\right)$, one is led to the straightforward conclusion of a negligible role played by convective flow of thermovibrational nature in comparison with Marangoni flow for $\varpi>\mathrm{O}\left(10^{3}\right)$ (which, in turn, implies that the defects displayed by PAS in this range of frequencies should be entirely ascribed to the direct influence of gjitters on particle motion via eq. 14).

For $\varpi \leq \mathrm{O}\left(10^{2}\right)$, however, as explained before, one should expect the oscillatory part of the gjitterinduced convective velocity field to be dominant, with the value of $\mathrm{Ra}_{\mathrm{v}}$ no longer providing a relevant characterization of the flow intensity.

In practice, it is known that in this range of frequencies $\operatorname{Ra}_{\omega}=\gamma \beta / \operatorname{Pr}$ should be assumed as a more relevant characteristic number (in addition, for $\varpi \leq \mathrm{O}\left(10^{2}\right)$, as in classical flows of gravitational nature, the amplitude of the oscillatory part of velocity is expected to scale according to $\left.\left(\operatorname{Ra}_{\omega}\right)^{1 / 2}\right)$.

For $\varpi=10^{2}$, in particular, the values taken by this nondimensional number are $\operatorname{Ra}_{\omega} \cong 250$ for $\gamma=10^{5}$ and $\operatorname{Ra}_{\omega} \cong 2500$ for $\gamma=10^{6}$; which indicates that the influence of gjitters-induced convective flow should be considered significant in comparison with Marangoni convection for $\gamma \geq \mathrm{O}\left(10^{6}\right)$ and negligible for $\gamma<\mathrm{O}\left(10^{6}\right)$.

To assess the validity of these arguments, we have plotted the temperature as a function of time measured at a generic point of the liquid bridge $(r=R / L, z=0.75$ and $\varphi=\pi)$ for different cases so as to appreciate the possible departures of such curves from the ideal behavior displayed by the system in the absence of imposed vibrations.

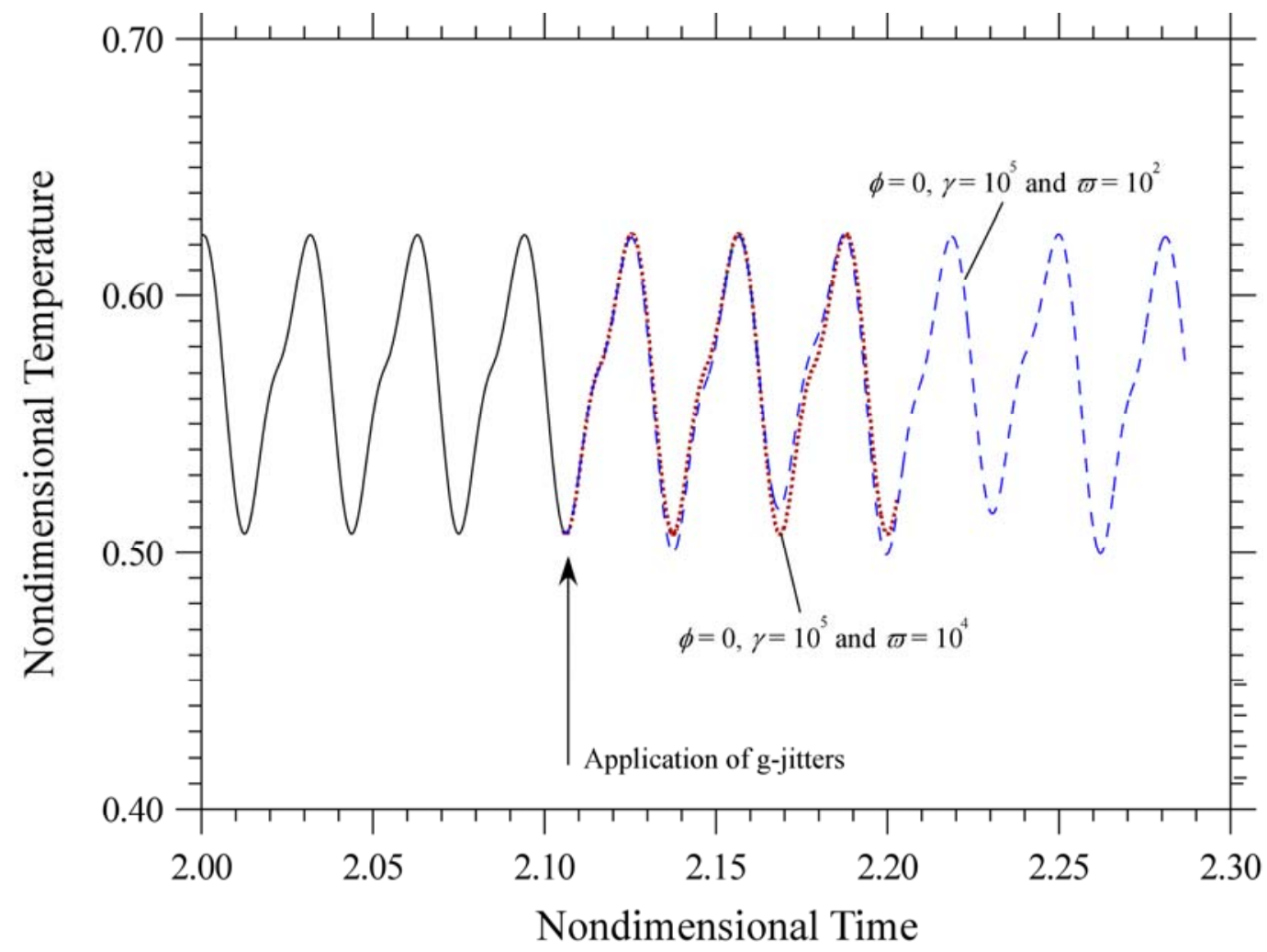

Figure 9: Temperature as a function of time measured at $r=R / L, z=0.75$ and $\varphi=\pi$ for different cases: no gjitter (solid line), $\phi=0, \gamma=10^{5}, \varpi=10^{2}$ (dashed line), and $\gamma=10^{5}, \varpi=10^{4}$ (dotted line). 
In line with our general expectations, Fig. 9 shows that the influence of considered gjitters ( $\phi=0$ and $\gamma=10^{5}$ ) on the underlying traveling wave is more or less negligible for all frequencies considered (some departure of the curve from the no-gjitters conditions is appreciable only for $\varpi \leq 10^{2}$, but changes in amplitude and frequency are very limited).

The analogous behavior for $\phi=0$ and $\gamma=10^{6}$ is summarized in Fig. 10.

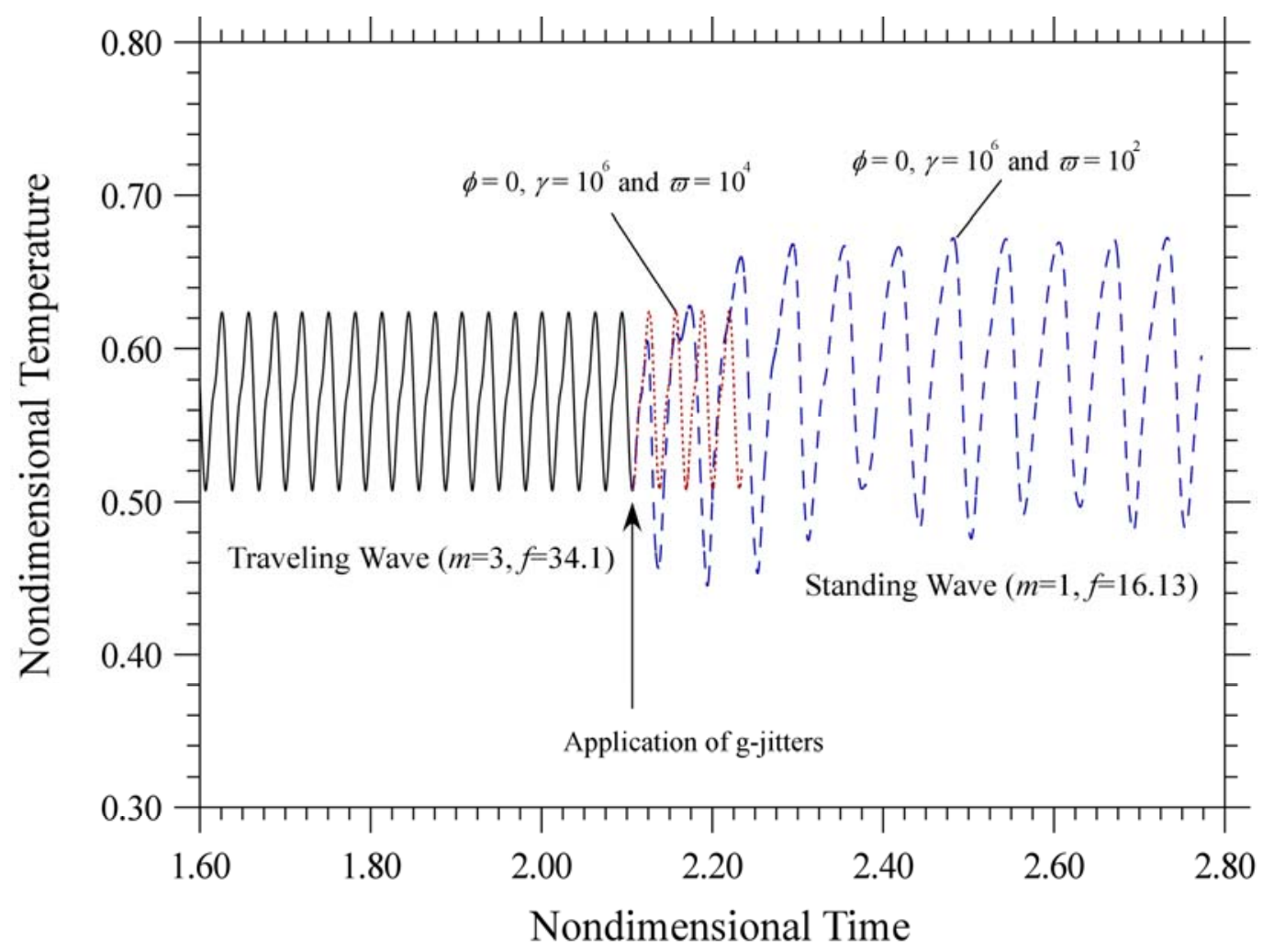

Figure 10: Temperature as a function of time measured at $r=R / L, z=0.75$ and $\varphi=\pi$ for different cases: no gjitter (solid line), $\phi=0, \gamma=10^{6}, \varpi=10^{2}$ (dashed line), and $\gamma=10^{6}, \varpi=10^{4}$ (dotted line).

Yet as expected, a clear change in the spatio-temporal flow structure can be observed for $\varpi=10^{2}$, as witnessed by the changes in amplitude and frequency of the related curve (while the curve for $\varpi=10^{4}$ still displays no appreciable modifications with respect to the case of no gjitters).

The structure and temporal organization of the flow field for these conditions $\left(\gamma=10^{6}\right.$ and $\left.\varpi=10^{2}\right)$ are shown in Figs. 11 and 12, respectively.

Interestingly, from these figures it can be inferred that the effect of gjitters on Marangoni flow does not determine solely a variation in the temperature oscillation amplitude and related frequency (changing from $\mathrm{f}=34.1$ to $\mathrm{f}=16.13$, see Fig. 10), as they also causes a decrease in the wavenumber of the supercritical Marangoni flow from $\mathrm{m}=3$ (no gjitters) to $\mathrm{m}=1$ (Fig.11). 


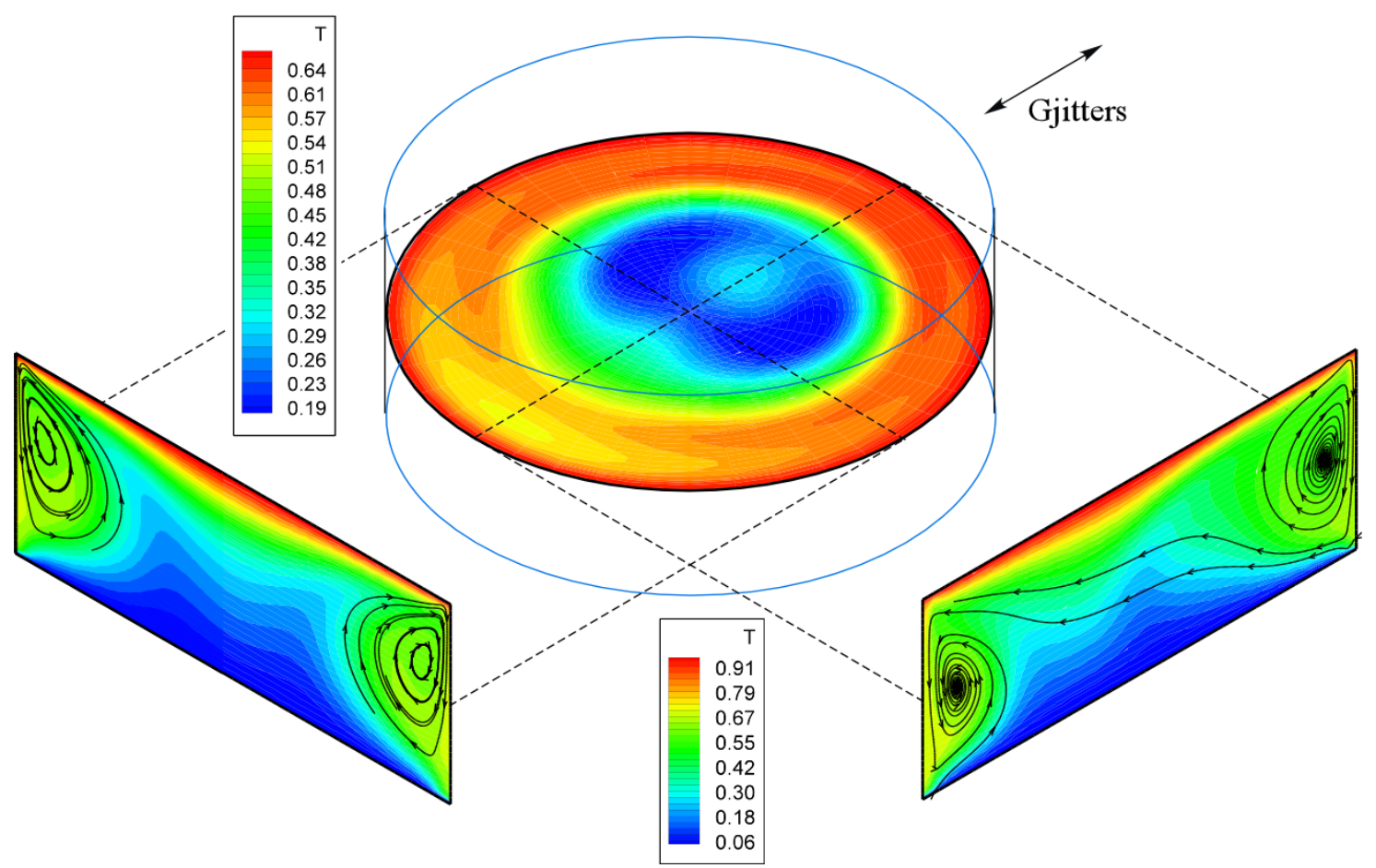

Figure 11: Snapshot of 3D thermo-fluid-dynamic field for $\phi=0, \gamma=10^{6}, \varpi=10^{2}$ (the flow looks like a standing wave with $\mathrm{m}=1$ ).
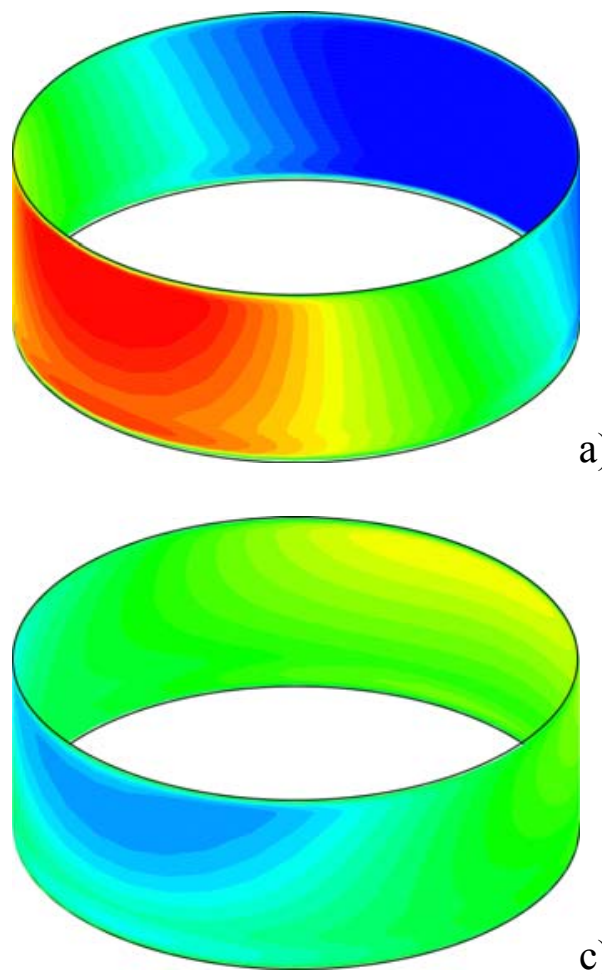

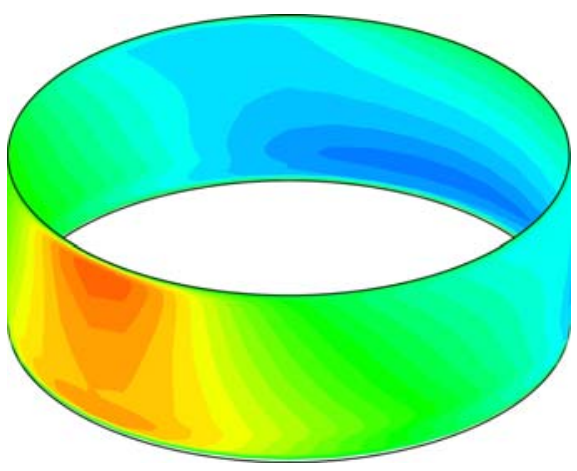

b)

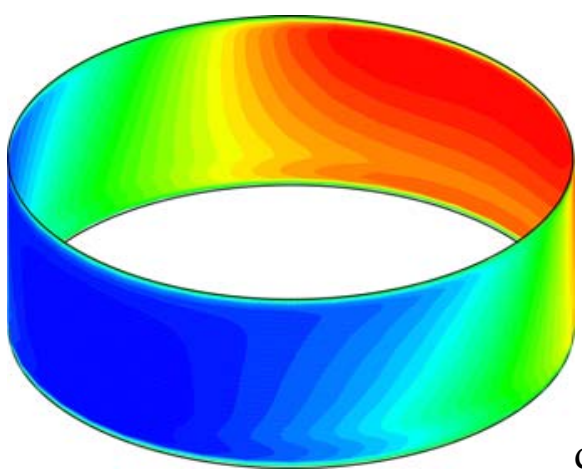

d)

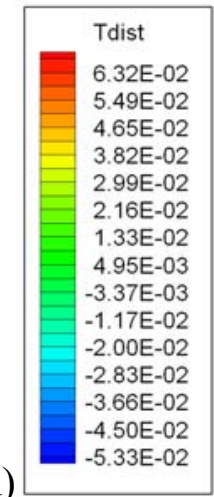

Figure 12: Snapshots of surface disturbance temperature distribution during half a period of oscillation for the same conditions considered in Fig. 11. They show the typical dynamics of a $\mathrm{m}=1$ standing wave (with disturbances at fixed azimuthal positions, growing, shrinking and changing sign in time; for the present case, in particular, it can be seen that the two pulsating nodes of opposite sign are perfectly aligned with the direction of imposed vibrations). 
What is even more remarkable (this is illustrated by Fig. 12), the waveform is no longer a traveling wave (which of PAS represents a necessary prerequisite, see Lappa, 2013b); the original propagation of the thermofluid-dynamic disturbance in the azimuthal direction, in fact, is replaced by hot and cold nodes at fixed azimuthal positions pulsating in time (i.e. a standing wave).

These numerical simulations prove, therefore, that PAS prevention at $\gamma=10^{6}$ in the low-frequency part of the spectrum should be ascribed primarily to the influence of gjitters on the underlying supercritical Marangoni flow rather than to their effect on the particle motion per se.

Let us recall that, as clarified in earlier studies (Schwabe and Mizev, 2011 and references therein; Lappa, 2013b), PAS can emerge in a given flow as closed lines rotating in the azimuthal direction as apparently solid items, provided specific conditions are established (which are the existence of at least one roll with toroidal structure and a disturbance traveling in the azimuthal direction having the characteristics of a wave). The transition from the initial $m=3$ traveling wave to $m=1$ standing wave, therefore, destroys the necessary pre-conditions on which PAS can be formed.

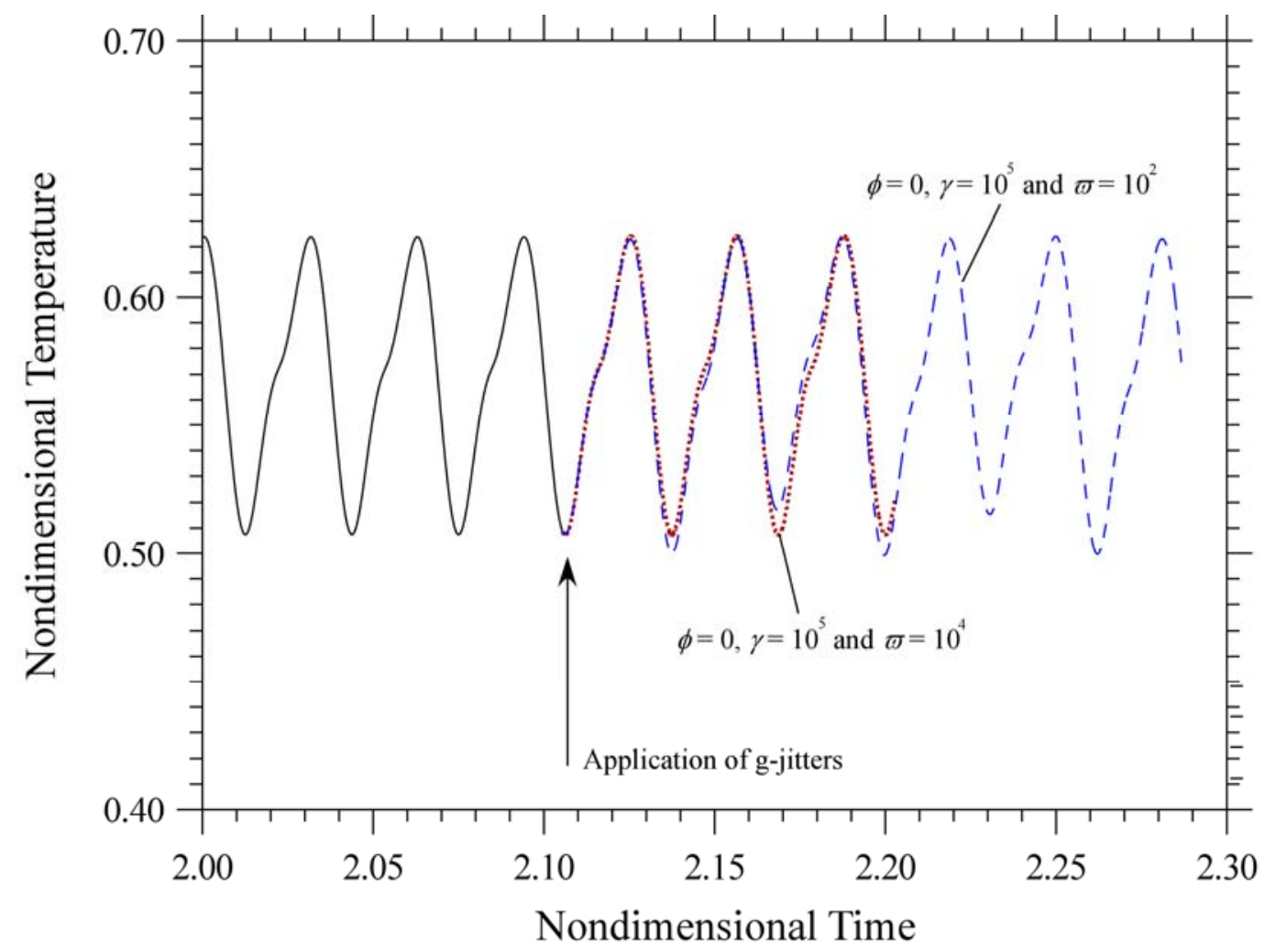

Figure 13: Temperature as a function of time measured at $r=R / L, z=0.75$ and $\varphi=\pi$ for different cases: no gjitter (solid line), $\phi=90, \gamma=10^{6}, \varpi=10^{2}$ (dashed line), and $\gamma=10^{6}, \varpi=10^{4}$ (dotted line).

Figure 13 provides an overview of the system response to the application of gjitters in the other situation in which gjitters are parallel to the axis of the liquid bridge $\left(\phi=0\right.$ and $\left.\gamma=10^{6}\right)$.

According to this figure, the influence of axial gjitters seems to be rather limited regardless of whether $\varpi \leq \mathrm{O}\left(10^{3}\right)$ or $\varpi>\mathrm{O}\left(10^{3}\right)$, which may be seen as an apparently puzzling results in the light of the scaling arguments provided before. 
In practice, for relatively high frequencies, these findings are yet a consequence of the thermovibrational theory (as we have seen earlier, the value of the vibrational Rayleigh number is very small). In addition to the argument based on the small value of the relevant characteristic number, one should also keep in mind that according to the Gershuni's theory, in the specific case of gjitters and temperature gradient acting along the same direction, the thermovibrational effect is expected to be very weak (Kamotani et al., 1981; Monti et al., 2001).

For relatively small frequencies, where the Gershuni's theory is no longer valid and in general the influence of gjitters should not be negligible, the absence of noticeable gjitter-induced flow, however, can be still ascribed to the considered direction of vibrations (in this case parallel to the imposed temperature gradient). As an example of existing studies on this subject, we may consider Hirata et al. (2001), who investigated numerically the canonical reference case of a 2D square cell in microgravity conditions. Assuming a liquid with $\operatorname{Pr}=7$ that is very close to that considered in the present work, they found the threshold for the onset of convection of vibrational nature in the case of gjitters parallel to the imposed temperature gradient to increase dramatically for increasing values of $\varpi$ (similar results were also obtained by Biringen and Danabasoglu, 1990). In particular, the value of $\mathrm{Ra}_{\omega}$ required for the onset of periodic convection was found to be $\cong 1.5 \times 10^{4}$ for $\varpi=10$, $\cong 3 \times 10^{4}$ for $\varpi=10^{2},>1 \times 10^{5}$ for $\varpi=10^{3}$, which are an order of magnitude larger than that in the present case $\left(\operatorname{Ra}_{\omega} \cong 2500\right.$ for $\left.\gamma=10^{6}\right)$.

We can therefore conclude that, for both cases of low and high frequencies, when the gjitters are parallel to the axis of the liquid bridge they are not able to influence significantly the supercritical Marangoni flow (therefore, they are limited to exerting their influence on PAS essentially as body forces acting directly on particles).

\subsection{Coloured Noise}

Until now we have considered monochromatic noise, i.e. inertial disturbances whose realization is a single periodic function of angular frequency $\varpi$. The goal of this final section is to provide a way of properly understanding more realistic situations, and to suggest approaches that might be used to analyse both observations and numerical solutions when the complete multi-frequency problem is considered.

Given the intrinsic nonlinear nature of the phenomena under analysis, it needs no demonstration that perturbations of velocity and temperature induced by distinct types of forcing will not just superpose linearly. For this reason, a straightforward generalization of the findings for monochromatic g-jitters to the more general "coloured" situation involving many distinct disturbances with different amplitudes, frequencies and directions, is not possible (Thomson et al., 1997). The problem is far more complex and would require its own separate exhaustive (parametric) treatment. Although, we will not attempt a complete solution here, however, some potentially useful indications are provided in this final section about the mechanism expected to be the most dangerous (i.e. the prevailing disturbance) for PAS formation. For the sake of simplicity and conciseness, in particular, we concentrate on a coloured inertial disturbance located in the transition 
region shown in both Figs 3 and 6, consisting of a discrete set of accelerations all with the same direction $(\phi=0$ or $\phi=\pi / 2)$, fixed amplitude and frequency ranging in the interval $5 \times 10 \leq \omega \leq 5 \times 10^{4}$. From a purely mathematical standpoint, this is implemented by replacing the quantity $\gamma \sin (\varpi t)$ in all relevant equations with the sum

$\sum_{j=1}^{4} \gamma_{j} \sin \left(\varpi_{j} t\right) \quad$ where $\quad \gamma_{j}=10^{5}$ and $\varpi_{j}=5 \times 10^{j}$

The results, shown in Fig. 14, provide evidence that (as one may expect on the basis of physical arguments), the patterns emerging under the combined influence of accelerations with comparable amplitudes, but different frequencies, display qualitatively all of the typical defects (as discussed in Sect. 3.1 and 3.2) which would be induced separately by each component of the spectrum. As anticipated, however, the most remarkable outcome of these simulations is the insight they give into the "most detrimental" effect of a coloured gjitter (in terms of departure at various spatial scales from the stationary pattern that the system should ideally maintain in the frame of reference rotating with the traveling wave), which seems to be produced essentially by its low-frequency components. This is clearly witnessed by the strongly asymmetric nature of the emerging pattern and the relatively large number of particles escaping the PAS attracting influence for $\phi=0$ (which is reminiscent of asymmetric and/or fringed PAS, compare, e.g., Fig. 14a with Fig. 4b and/or Fig. 4d, respectively) and the strongly time-dependent nature of the structures formed by the accumulation of particles in the $\phi=\pi / 2$ case (which is reminiscent of P-PAS, compare, e.g., Fig. 14b and Fig. 8a).

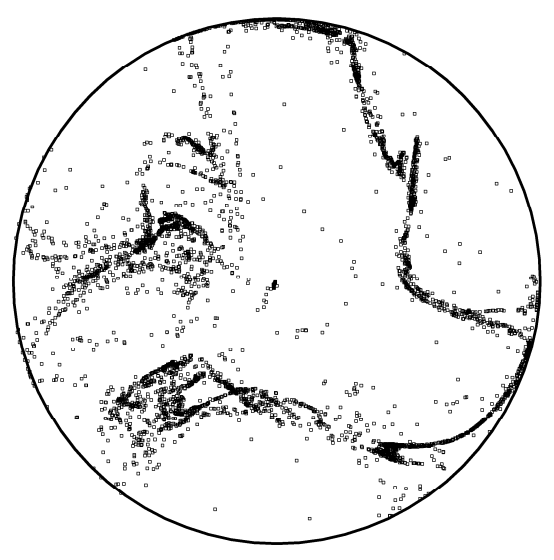

a)

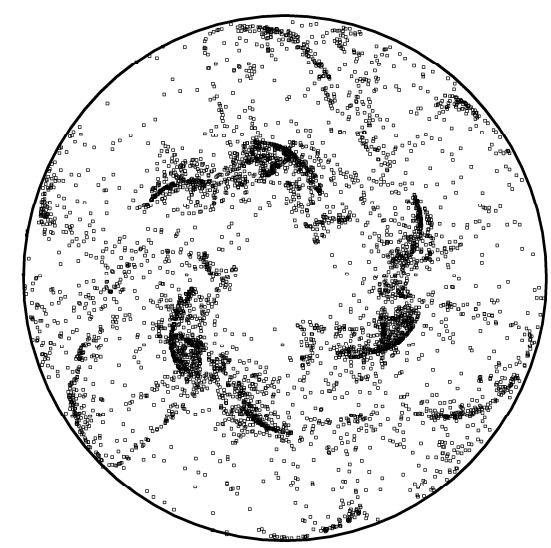

b)

Figure 14: Snapshot of PAS for coloured gjitter with fixed amplitude $\gamma=10^{5}$ and frequency ranging in the interval $5 \times 10 \leq \varpi \leq 5 \times 10^{4}$ according to eq. (24): a) $\phi=0$ (equatorial gjitter), b) $\phi=\pi / 2$ (axial gjitter).

\section{Conclusions}

The collective behaviors of solid particles dispersed in supercritical Marangoni flow have been investigated by direct numerical solution of the governing flow-field (Navier-Stokes-Boussinesq) 
equations in combination with a specific particle-tracking method accounting for particle motion under the influence of inertia and viscous drag. Attention has been concentrated on a traveling-wave solution, which of Marangoni convection represents a well-known testbed.

As shown by experiments and confirmed by numerical simulations, if specific conditions are satisfied, particles, initially uniformly spaced in the liquid, undergo segregation and form a regular three-lobe-shaped one-dimensional circuit, which behaves apparently as a solid wire rotating with the angular frequency of the hydrothermal wave.

This relatively simple description, however, is no longer applicable as soon as complications, such as external periodic perturbations of inertial nature are added to the considered problem.

These perturbations can prevent PAS formation at all. In general, however, there exist, between the limiting purely regular and irregular particle distributions in space (and/or time), numerous intermediate situations. One of the most interesting outcomes of the present work is that these dynamics and the related transitional stages are largely determined by a sort of dialectics between the tendency of particle inertia to support solid phase self-organization (in combination with the supercritical Marangoni flow and its traveling thermo-fluid-dynamic disturbance) and to counteract, at the same time, this process by promoting disorder and chaos (as a result of the application of gravity disturbances).

The numerical simulations have shown how, in general, the behavior at a large scale of the system and the resulting topological and morphological properties of the PAS arise from detailed structures and interdependencies on different scales. In such a context, some effort has been directed to separate expressly on such scales the defects which characterize the pattern and create its recognizable identification. In particular, a possible categorization of the resulting particle structures has been introduced on the basis of the spatial and temporal scale of the prevailing disturbance and its typical manifestation in the reference frame rotating at the same angular velocity of the traveling thermo-fluid-dynamic wave.

We have found that both large and small scale defects, appearing as asymmetries in the shape of the PAS and turbulent displacements of localized sets of particles, respectively, can affect PAS dynamics at intermediate values of the vibrations nondimensional frequency. A more precise assessment of the impact of gjitters frequency $(\varpi)$ and amplitude $(\gamma)$, however, has revealed that for a fixed value of $\gamma$ a first regime exists where the amplitude of the large-scale disturbance is almost constant and independent of $\varpi$. As this frequency attains progressively larger values, the large-scale disturbance is taken over by the emergence of a new class of disturbances on a smaller scale, consisting of a cyclic departure of particles from the purely one-dimensional path that would be maintained in the ideal gjitter-less conditions. An additional increase in $\varpi$ tends to suppress also these dynamics leading to a full recovery of the classical unperturbed PAS. By contrast, increases in $\gamma$ at a constant value of $\varpi$ cause, in general, an increase in the amplitude of both small and large scale defects until no recognizable association of particles becomes possible above a certain $\gamma(\varpi)$ (no PAS region).

The observed ensemble phenomena have been explained in the framework of a theory that has its root in a long tradition of past studies devoted to phenomena of inertial particle clustering in 
synergy with dimensional arguments and existing theories on the effect of periodic accelerations on (multiphase and non-isothermal) fluid systems.

Perhaps the best example of the conflicting roles played by particle inertia in the considered problem (on the one hand it leads to particle association via the phase-locking mechanism, and on the other hand inertia disturbs their ordered aggregation through gjitters-induced motions) is given by the case in which the vibrations are parallel to the axis of the liquid bridge. In this situation, at moderate values of $\gamma$, a range of vibration frequencies exists in a certain neighborhood of the frequency of the traveling wave where the particles give rise during one period of gjitter oscillation to the birth and death of several recognizable geometric shapes (ranging from multi-armed windmills to multi-sided star-shaped polygons and even fractal-like items), all alternating during one period of oscillation so as to create the illusion of a "pulso-rotating" pattern in the fixed (laboratory) reference frame.

Although the dynamics of particles at intermediate values of $\gamma$ seem to be consistent with the expected scenario predicted by scaling relationships determined in the past for relatively simple and idealized fluid-particle systems, we have found that, in addition to the influence exerted by periodic accelerations directly on particle motions (as a consequence of the solid-fluid density difference), there are other physical connections in which the vibrational disturbances are important. We refer, in particular, to the flow of thermovibrational nature originating from the application of periodic accelerations to a non-isothermal fluid.

These flows must be necessarily invoked to explain correctly the mechanism of PAS prevention in some circumstances (the region of high $\gamma$ and small $\varpi$ ). Along these lines, the numerical simulations have demonstrated that, when the considered vibrations are perpendicular to the axis of the liquid bridge, the thermovibrational component of the oscillatory flow can cause a suppression of the necessary conditions for the existence of PAS by forcing the supercritical Marangoni flow to replace the azimuthally traveling thermo-fluid-dynamic disturbance with a "standing wave".

Finally, when the representation of the considered inertial disturbance is enriched so as to mimic the case of coloured noise, the simulations indicate that the "most detrimental" effect on the resulting pattern is produced essentially by the acceleration components lying in the low-frequency part of the spectrum.

The sensitivity of these phenomena to vibrations/gjitters and ensuing variety of possible spatiotemporal behaviors may be regarded as a further example of the richness and complexity of this specific subject which makes the study of PAS formation a subject of great interest to the community of theoretical physicists and a truly interdisciplinary topic. Indeed, the peculiar path that the study of this problem has taken over recent years may be regarded as another example of how several decades of explorations in diverse areas such as pattern recognition, Marangoni flow, thermovibrational convection, multiphase flow and more recently theories of inertial particle clustering, has honed the ability of researchers to identify and apply common sets of principles across various disciplines. 


\section{ACKNOWLEDGMENTS}

This work is supported by the Italian Space Agency (ASI) in the framework of the JEREMI (Japanese and European Research Experiment on Marangoni Instabilities) Project (ASI contract I/048/11/0) for the preparation and execution of an experiment onboard the International Space Station (ISS).

\section{REFERENCES}

Ahadi A.H. and Saghir M.Z., (2012), Quasi Steady State Effect of Micro Vibration from Two Space Vehicles on Mixture During Thermodiffusion Experiment, Fluid Dyn. Mater. Process., 8(4),. 397-422.

Alexander J.I.D., (1990), Low gravity experiment sensitivity to residual acceleration: a review, Microgravity Sci. and Tech., I (3), 52-68.

Atkinson K.A., An Introduction to Numerical Analysis (2nd ed.), (John Wiley \& Sons, New York, 1989).

Babiano A., Cartwright J. H. E., Piro O. and Provenzale A., (2000), Dynamics of a small neutrally buoyant sphere in a fluid and targeting in Hamiltonian systems, Phys. Rev. Lett., 84, 57645767.

Baird M. H. I., Senior M. G., and Thompson R. J., (1967), Terminal Velocities of Spherical Particles in a Vertically Oscillating Liquid, Chem. Eng. Sci., 22, 551-558.

Balboa Usabiaga F., Pagonabarraga I., and Delgado-Buscalioni R., (2013), Inertial coupling for point particle fluctuating hydrodynamics, J. Comp. Phys., 235, 701-722.

Balkovsky E., Falkovich G., and Fouxon A., (2001), Intermittent Distribution of Inertial Particles in Turbulent Flows, Phys. Rev. Lett. 86, 2790-2793.

Benczik I. J., Toroczkai Z., and Tél T., (2002), Selective Sensitivity of Open Chaotic Flows on Inertial Tracer Advection:Catching Particles with a Stick, Phys. Rev. Lett., 89 (16), 164501

Biringen S. and Danabasoglu G., (1990), Computation of convective flows with gravity modulation in rectangular cavities, J. Thermophys., 4, 357-365.

Bothe D., Kröger M. and Warnecke H.-J. (2011), A VOF-Based Conservative Method for the Simulation of Reactive Mass Transfer from Rising Bubbles, Fluid Dyn. Mater. Process., 7(3), 303-316

Busse F. H., Pfister G., Schwabe D., (1998), Formation of Dynamical Structures in Axisymmetric Fluid Systems, in Evolution of Spontaneous Structures in Dissipative Continuous Systems, Lecture Notes in Physics Volume 55, 1998, pp 86-126

Carotenuto L., Piccolo C., Castagnolo D., Lappa M., Garcì-Ruiz J.M., (2002), Experimental observations and numerical modelling of diffusion-driven crystallisation processes, Acta Crystallographica D, 58, 1628-1632.

Coimbra F.M. and Rangel R.H. (2001), Spherical particle motion in harmonic stokes flows, AIAA J, 39(9), 1673-1682.

Coimbra C.F.M., L'Esperance D., Lambert R.A., (2004), An experimental study on stationary history effects in high-frequency stokes flows, J. Fluid Mech. 504, 353-363.

Derksen J.J. and Eskin D., (2011), Flow-Induced Forces in Agglomerates, Fluid Dyn. Mater. Process., 7(4), 341-356. 
Di Carlo D., Edd J. F., Humphry K. J., Stone H.A., and Toner M., (2009), Particle Segregation and Dynamics in Confined Flows, Phys. Rev. Lett., 102, 094503.

Druzhinin O.A., and Ostrovsky L.A., (1994), The Influence of Basset Force on Particle Dynamics in Two Dimensional Flows, Physica D, 76, 34-43.

Dyko M. P. and Vafai K., (2007), Effects of gravity modulation on convection in a horizontal annulus, Int. J. heat Mass Transfer, 50, 348-360.

Ellison J., Ahmadi G., Regel L. and Wilcox W., (1995), Particle motion in a liquid under g-jitter excitation, Microgravity Sci. and Tech., 8, 140- 147.

Esmaeeli A., (2005), Phase Distribution of Bubbly Flows under Terrestrial and Microgravity Conditions, Fluid Dyn. Mater. Process., 1(1), 63-80.

Esmaeeli, A. and Tryggvason, G. (1998), Direct Numerical Simulations of Bubbly Flows. Part ILow Reynolds Number Arrays, J. Fluid Mech., 377, 313-345.

Gershuni G.Z., Lyubimov D.V., Lyubimova T.P., Roux, B., (1994), Convective flows in a liquid bridge under the influence of high frequency vibrations, Microgravity Q., 4 (2), 113-122.

Gershuni G.Z. and Zhukhovitskii E.M., (1981), Convective instability of a fluid in a vibration field under conditions of weightlessness, Fluid Dynamics, 16(4), 498-504.

Gershuni G.Z., Zhukhovitskii E.M., (1986), Vibrational thermal convection in zero gravity, Fluid. Mech. Sov. Res., 15(1), 63-84.

Haller G. and Sapsis T., (2012), Lagrangian coherent structures and the smallest finite-time Lyapunov exponent, Chaos, 21(2), 023115.

Hassan S., Lyubimova T.P., Lyubimov D.V., Kawaji M., (2006a), Motion of a sphere suspended in a vibrating liquid-filled container, J. Appl. Mech., 73, 72-78.

Hassan S., Lyubimova T. P., Lyubimov D. V., Kawaji M., (2006b), Effects of vibrations on particle motion near a wall: Existence of attraction force, Int. J. Multiphase Flow, 32 (9), 1037-1054.

Hassan S. and Kawaji M., (2008), The Effects of Vibrations on Particle Motion in a Viscous Fluid Cell, J. Appl. Mech., 75, 031012 [7 pages].

Herringe R.A., (1977), A study of particle motion induced by two-dimensional liquid oscillations, Int. J. Multiphase Flow, 3(3), 243-253

Hirata K., Sasaki T., Tanigawa H., (2001), Vibrational effects on convection in a square cavity at zero gravity, J. Fluid Mech., 445, 327-344.

Hjelmfelt A. T., and Mockros L. F., (1966), Motion of Discrete Particles in a Turbulent Fluid, Appl. Sci. Res., 16(1), 149-161.

Homma S., Yokotsuka M., Tanaka T., Moriguchi K., Koga J. and Tryggvason G., (2011), Numerical Simulation of an Axisymmetric Compound Droplet by Three-Fluid Front-Tracking Method, Fluid Dyn. Mater. Process., 7(3), 231-240.

Houghton G., (1961), The behaviour of particles in a sinusoidal vector field, Proc. Roy. Soc. A 272 , 33-43.

Ikeda S., (1989), Fall Velocity of Single Spheres in Vertically Oscillating Fluids, Fluid Dyn. Res., 5, 203-216.

Kamotani Y., Prasad A. and Ostrach S., (1981), "Thermal convection in an enclosure due to vibrations aboard spacecraft", AIAA J. 19, 511-516.

Kleckner D. and Irvine W. T. M. (2013), Creation and dynamics of knotted vortices, Nature Physics, doi:10.1038/nphys 2560

Kozlov V.G., Ivanova A.A. and Evesque P., (2006), Block stratification of sedimenting granular matter in a vessel due to vertical vibration, Fluid Dyn. Mater. Process., 2(3), 203-210. 
Kuhlmann H. C. and Muldoon F. H., (2012), Understanding particle accumulation structures (PAS) in thermocapillary liquid bridges, JASMA, 29(2), 64-98.

Langbein D., (1991), Motion of Ensembles of Spherical Particles in a Fluid Due to g-jitter, Adv. Space Res., 11(7), 189-196.

Lappa M., (2003a), Three-dimensional numerical simulation of Marangoni flow instabilities in floating zones laterally heated by an equatorial ring, Phys. Fluids, 15 (3), 776-789.

Lappa M., (2003b), Growth and Mutual Interference of Protein Seeds under reduced gravity conditions, Physics of Fluids, 15 (4), 1046-1057.

Lappa M., (2004), Combined effect of volume and gravity on the three-dimensional flow instability in non-cylindrical floating zones heated by an equatorial ring, Phys. Fluids, 16 (2), 331-343.

Lappa M., (2005), Assessment of VOF Strategies for the analysis of Marangoni Migration, Collisional Coagulation of Droplets and Thermal wake effects in Metal Alloys under Microgravity conditions, Computers, Materials \& Continua, 2(1), 51-64.

Lappa M., (2006), Oscillatory convective structures and solutal jets originated from discrete distributions of droplets in organic alloys with a miscibility gap, Phys. Fluids, 18 (4), 042105.

Lappa M., Thermal Convection: Patterns, Evolution and Stability (John Wiley \& Sons, Chichester, England, 2010).

Lappa M., Rotating Thermal Flows in Natural and Industrial Processes (John Wiley \& Sons, Chichester, England, 2012).

Lappa M., (2011), A theoretical and numerical multiscale framework for the analysis of pattern formation in protein crystal engineering, Int. J. Multiscale Comp. Eng., 9 (2), 149-174.

Lappa M., (2013a), Assessment of the role of axial vorticity in the formation of Particle Accumulation Structures in supercritical Marangoni and hybrid thermocapillary-rotationdriven flows, Phys. Fluids, 25(1), 012101.

Lappa M., (2013b), On the Existence and Multiplicity of One-dimensional Solid Particle Attractors in Time-dependent Rayleigh-Bénard Convection, Chaos, 23(1), 013105. DOI: $10.1063 / 1.4773001$

Lappa M., Carotenuto L., (2003), Effect of convective disturbances induced by g-jitter on the periodic precipitation of lysozyme, Microgravity Sci. Tech., XIV/2, 41-56.

Lappa M., Castagnolo D., Carotenuto L., (2002), Sensitivity of the non-linear dynamics of Lysozyme 'Liesegang Rings' to small asymmetries, Physica A, 314/1-4, 623-635.

Lappa M.. and Savino R., (1999), Parallel solution of the three-dimensional Marangoni flow instabilities in liquid bridges, Int. J. Num. Meth. Fluids, 31(6), 911-925.

Lappa M. and Savino R., (2002), 3D analysis of crystal/melt interface shape and Marangoni flow instability in solidifying liquid bridges, J. Comput. Phys., 180 (2), 751-774.

Lappa M., Piccolo C., (2004), Higher modes of Mixed Buoyant-Marangoni unstable convection originated from a droplet dissolving in a liquid/liquid system with miscibility gap, Phys. Fluids, 16(12), 4262-4272.

Lappa M., Piccolo C., Carotenuto L., (2004), Mixed buoyant-Marangoni convection due to dissolution of a droplet in a liquid-liquid system with miscibility gap, European Journal of Mechanics/B Fluids, 23/5, 781-794.

Lappa M., Yasushiro S., Imaishi N., (2003), 3D numerical simulation of on ground Marangoni flow instabilities in liquid bridges of low Prandtl number fluid, Int. J. Num. Meth. Heat Fluid Flow, 13 (3), 309-340. 
Lappa M., Savino R. and Monti R., (2000), Influence of buoyancy forces on Marangoni flow instabilities in liquid bridges, Int. J. Num. Meth. Heat Fluid Flow, 10 (7), 721-749.

Lighthill M. J., (1954), The Response of Laminar Skin Friction and Heat Transfer to Fluctuations in the Stream Velocity, Proc. R. Soc. London, Ser. A, 224, 1-23.

Lizée A. and Alexander J.I.D., (1997), Chaotic thermovibrational flow in a laterally heated cavity, Phys. Rev. E, 56, 4152-4156.

Mark A., Rundqvist R. and Edelvik F., (2011), Comparison Between Different Immersed Boundary Conditions for Simulation of Complex Fluid Flows, Fluid Dyn. Mater. Process., 7(3), 241-258

Maxey M. R. and Riley J. J., (1983), Equation of motion for a small rigid sphere in a nonuniform flow, Phys. Fluids, 26, 883-889.

Maxey M.R., Patel B.K., Chang E.J., Wang L.-P., (1997), Simulations of dispersed turbulent multiphase flow, Fluid Dyn. Res., 20(1-6), 143-156.

Mei R., Lawrence J., and Adrian J., (1991), Unsteady Drag on a Sphere at Finite Reynolds Number With Small Fluctuations in the Free-Stream Velocity, J. Fluid Mech., 233, 613-631.

Melnikov D., Pushkin D., and Shevtsova V., (2011) Accumulation of particles in time-dependent thermocapillary flow in a liquid bridge. Modeling of experiments, Eur. Phys. J. Special Topics, 192, 29-39.

Michaelides E.E., (1997), Review - The transient equation of motion for particles, bubbles, and droplets, J. Fluids Eng., 119, 233-247.

Monti R., Savino R., Lappa M., (2001), On the convective disturbances induced by g-jitter on the space station, Acta Astronautica, 48 (5-12), 603-615.

Monti R., Savino R., Lappa M., (1998), Microgravity sensitivity of typical fluid physics experiment, presented at the 17 th Microgravity Measurements Group Meeting, Cleveland, Ohio, 24-26 March 1998, published in the meeting proceedings in NASA CP-1998-208414, 23, pp. 1-15 (ISSN: 0191-7811).

Parsa A. and Saghir M.Z., (2012), Fluid Flow Behavior of a Binary Mixture Under the Influence of External Disturbances Using Different Density Models, Fluid Dyn. Mater. Process., 8(1), $27-$ 50

Pasquero C., Provenzale A., and Spiegel E. A., (2003), Suspension and Fall of Heavy Particles in Random Two-Dimensional Flow, Phys. Rev. Lett., 91, 054502.

Pushkin D., Melnikov D., Shevtsova V., (2011), Ordering of Small Particles in One-Dimensional Coherent Structures by Time-Periodic Flows, Phys. Rev. Lett., 106, 234501.

Raju N. and Meiburg E., (1995), The accumulation and dispersion of heavy particles in forced twodimensional mixing layers. Part 2: The effect of gravity, Phys. Fluids, 7, 1241-1264.

Sapsis T. and Haller G., (2010), Clustering criterion for inertial particles in two-dimensional timeperiodic and three-dimensional steady flows, Chaos, 20, 017515.

Savino R., Lappa M., (2003), Assessment of the thermovibrational theory: application to g-jitter on the Space-station, Journal of Spacecraft and Rockets, 40 (2), 201-210.

Savino R., Paterna D., Lappa M., (2003), Marangoni flotation of liquid droplets, J. Fluid Mech., 479, 307-326.

Saw E. W., Shaw R. A., Ayyalasomayajula S., Chuang P. Y., and Gylfason A., (2008), Inertial Clustering of Particles in High-Reynolds-Number Turbulence, Phys. Rev. Lett., 100, 214501. 
Schwabe D. and Mizev A.I., (2011), Particles of different density in thermocapillary liquid bridges under the action of travelling and standing hydrothermal waves, Eur. Phys. J. Special Topics, 192, 13-27

Schwabe D., Mizev A. I., Tanaka S. and Kawamura H., (2006), Particle accumulation structures in time-dependent thermocapillary flow in a liquid bridge under microgravity, Microgravity Sci. Tech., 18(3-4), 117-127 .

Schwabe D., Mizev A.I., Udhayasankar M. and Tanaka S., (2007), Formation of dynamic particle accumulation structures in oscillatory thermocapillary flow in liquid bridges, Phys. Fluids, 19(7), 072102.

Shevtsova V., Mialdun A., Kawamura H., Ueno I., Nishino K., Lappa M., (2011), Onset of Hydrothermal Instability in Liquid Bridge. Experimental Benchmark, Fluid Dyn. Mater. Process., 7(1), 1-28.

Shevtsova V., Kuhlmann H., Montanero J.M., Nepomnyaschy A., Lappa M., Schwabe D., Matsumoto S., Nishino K., Ueno I., Yoda S., (2008), Preparation of Space experiment in the FPEF facility: Heat transfer at the interface in the systems with cylindrical symmetry, 26th International Symposium on Space Technology and Science, June 1-8 2008, Hamamatsu City (ISTS Proceedings).

Shevtsova V., Gaponenko Y. A. and Nepomnyashchy A., (2013), Thermocapillary flow regimes and instability caused by a gas stream along the interface, J. Fluid Mech., 714, 644-670.

Simic-Stefani S., Kawaji M. and Hu H., (2006), G-jitter induced motion of a protein crystal under microgravity, J. Cryst. Growth, 294, 373-384.

Srinivasan S. and Saghir M. Z., (2011), Impact of the Vibrations on Soret Separation in Binary and Ternary Mixtures, Fluid Dyn. Mater. Process, 7(2), 201-216.

Sun J., Carlson F.M., Regel L.L., Wilcox W.R., Lal R.B., Trolinger J.D., (1994), Particle motion in the Fluid Experiment System in Microgravity, Acta Astronautica, 34, 261-269

Tanaka S., Kawamura H., Ueno I., Schwabe D., (2006), Flow structure and dynamic particle accumulation in thermocapillary convection in a liquid bridge, Phys. Fluids, 18, 067103.

Thomson J.R., Casademunt J., Drolet F. and Vinals J., (1997), Coarsening of solid-liquid mixtures in a random acceleration field, Phys Fluids, 9(5), 1336-1343.

Tunstall E. B., and Houghton G., (1968), Retardation of Falling Spheres by Hydrodynamic Oscillations, Chem. Eng. Sci., 23, 1067-1081.

Uchiyama T., (2011), Grid-Free Vortex Method for Particle-Laden Gas Flow, Fluid Dyn. Mater. Process., 7(4), 371-388.

Ueno I., Ono Y., Nagano D., Tanaka S. and Kawamura H. (2000), Modal oscillatory structure and dynamic particle accumulation in liquid-bridge Marangoni convection, in '4th JSME-KSME Thermal Engineering Conference', JSME, Kobe, Japan

Ueno I., Abe Y., Noguchi K., and Kawamura H., (2008), Dynamic particle accumulation structure (PAS) in half-zone liquid bridge - Reconstruction of particle motion by 3-D PTV, Adv. Space Res., 41(12), 2145-2149.

Vojir D. J. and Michaelides E. E., (1994), The Effect of the History Term on the Motion of Rigid Spheres in a Viscous Fluid, Int. J. Multiphase Flow, 20, 547-556.

Yan Y., Shevtsova V., Saghir M.Z., (2005), Numerical study of low frequency g-jitter effect on thermal diffusion, Fluid Dyn. Mater. Process., 1(4), 315-328.

Yan Y., Jules K. and Saghir M.Z., (2007), Comparative Study of G-jitter Effect on Thermal Diffusion aboard the International Space Station, Fluid Dyn. Mater. Process., 3(3), 231-246. 
J. Fluid. Mech, Vol. 726, pp. 160-195, (2013)

Zaichik L., Alipchenkov V.M., and Sinaiski E.G., Particles in Turbulent Flows, 2008 WILEY-VCH Verlag GmbH \& Co. KGaA, Weinheim

Zinchenko A. Z. (1994), An efficient algorithm for calculating multiparticle thermal interaction in a concentrated dispersion of spheres, J. Comput. Phys., 111, 120-134.

Zohdi T.I., An Introduction to modeling and Simulation of Particulate Flows, SIAM, 2007. 